Aus der Klinik für Unfallchirurgie, Orthopädie und Plastische Chirurgie

(Prof. Dr. med. W. Lehmann)

der Medizinischen Fakultät der Universität Göttingen

\title{
Korrelation zwischen erwarteter und reeller Verlängerung bei extern gesteuerten magnetischen wirbelsäulenaufrichtenden Implantaten im Kindesalter
}

\author{
INAUGURAL-DISSERTATION \\ zur Erlangung des Doktorgrades \\ der Medizinischen Fakultät der \\ Georg-August-Universität zu Göttingen \\ vorgelegt von \\ Jasmin Grote (geb. Lachmann) \\ aus \\ Braunschweig
}

Göttingen 2019 
Dekan:

Referent/in

Ko-Referent/in:

Drittreferent/in:

Datum der mündlichen Prüfung:
Prof. Dr. rer. nat. H. K. Kroemer

Prof. Dr. med. A.-K. Hell

Prof. Dr. med. H.- C. Ludwig

Prof. Dr. mult. T. Meyer

12.03.2020 
Hiermit erkläre ich, die Dissertation mit dem Titel "Korrelation zwischen erwarteter und reeller Verlängerung bei extern gesteuerten magnetischen wirbelsäulenaufrichtenden Implantaten im Kindesalter" eigenständig angefertigt und keine anderen als die von mir angegebenen Quellen und Hilfsmittel verwendet zu haben.

Göttingen, den 10.07.2019 
Teile dieser Dissertation sind bereits publiziert worden:

\section{Publikation:}

Lorenz HM, Braunschweig L, Badwan B, Groenefeld K, Hecker MM, Tsaknakis K, Grote J, Hell AK (2018): High Correlation Between Achieved and Expected Distraction Using Magnetically Controlled Growth Rods (MCGR) With Rib to Pelvis Fixation in Pediatric Spine Deformity. J Pediatr Orthop 39, E334-E338 


\section{Inhaltsverzeichnis}

Abbildungsverzeichnis .................................................................................... III

Tabellenverzeichnis ......................................................................................... III

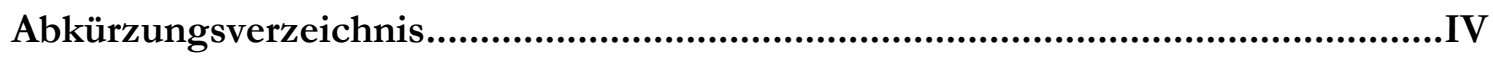

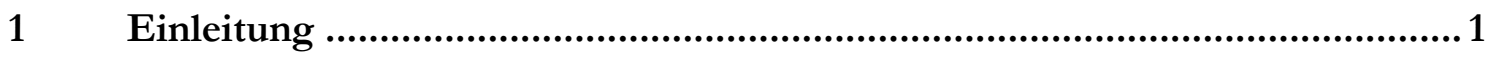

1.1 Implantate für kindliche Wirbelsäulendeformitäten .............................................................2

1.1.1 Vertical expandable prosthetic titanium rib (VEPTR) ..................................................................... 3

$1.2 \quad$ Nachteile bisheriger wachstumsfreundlicher Implantate .............................................................. 3

1.3 Extern zu kontrollierende magnetische Implantate ..................................................................

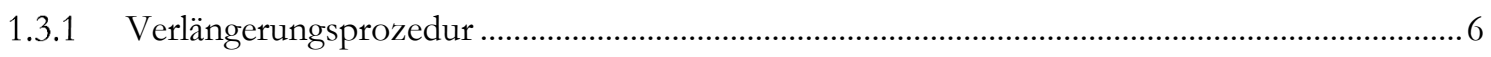

1.3.2 Probleme bei Verlängerungsprozeduren .........................................................................

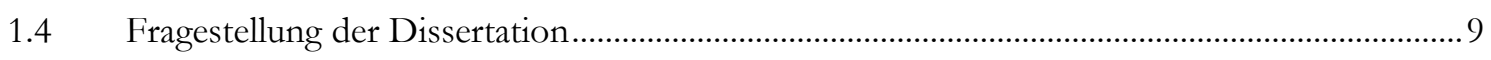

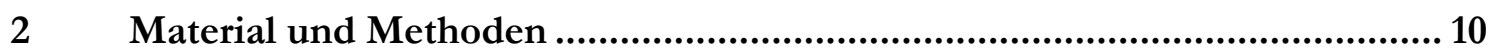

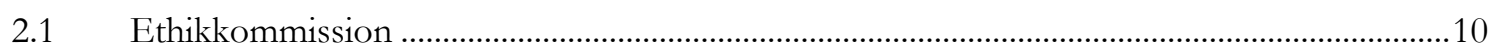

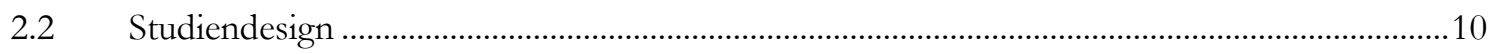

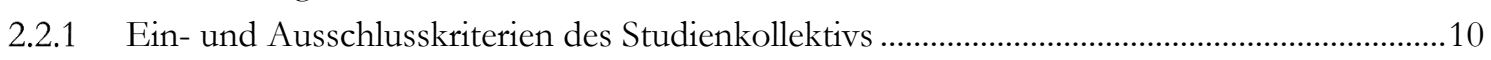

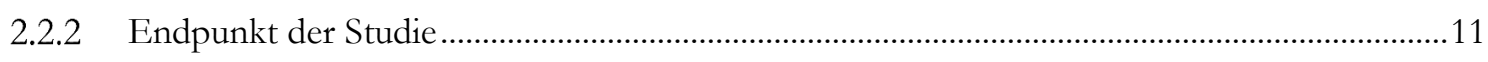

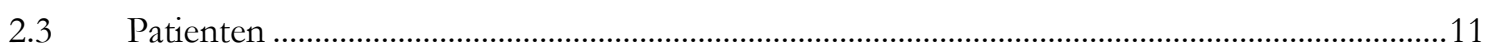

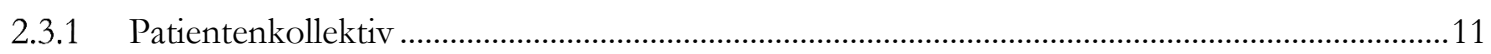

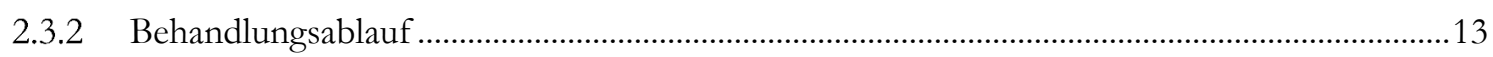

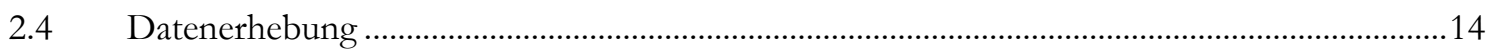

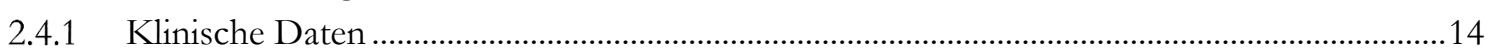

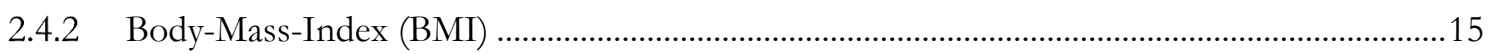

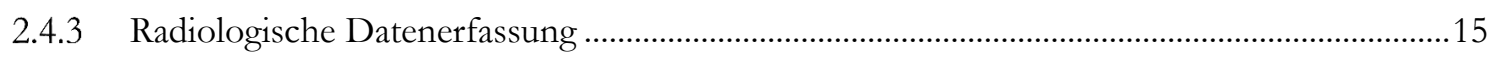

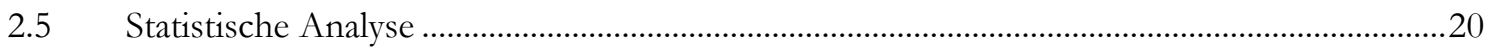

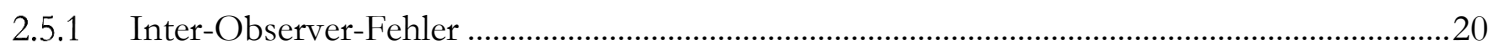

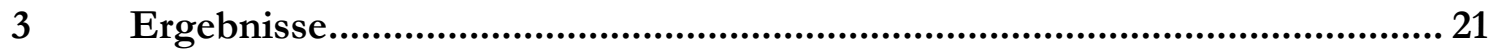

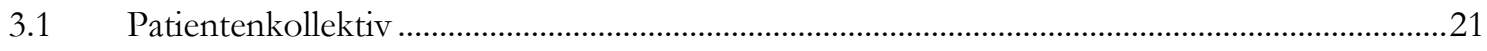

3.2 Radiologische Wirbelsäulendeformitätenparameter ...............................................................24

3.3 Erwarteter Implantatauszug im Vergleich zur tatsächlich gemessenen Implantatlänge

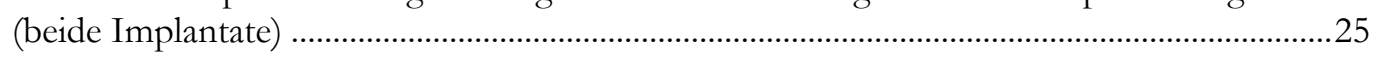

3.3.1 Unterschied zwischen dem konkaven und konvexen Implantat ..............................................2

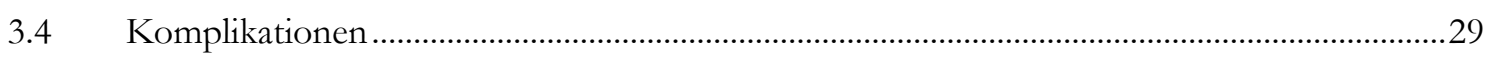

3.5 Korrelation des röntgenologisch ermittelten Implantatauszuges und des Cobb-Winkels bei Kindern mit Komplikationen .............................................................................................

3.6 Unbeabsichtigtes Implantat-Ein- oder Ausfahren zwischen den Verlängerungen...................31 


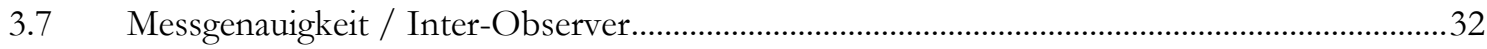

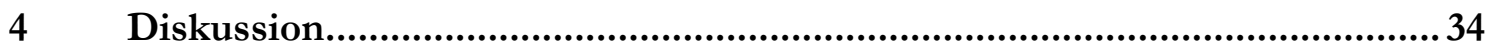

4.1 Vergleichende Analyse von erwarteter zur tatsächlichen Auszuglänge des MAGEC®-

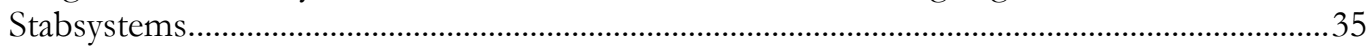

4.2 Vergleich von Stablänge und veränderte Wirbelsäulenlänge........................................................36

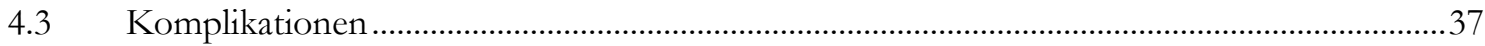

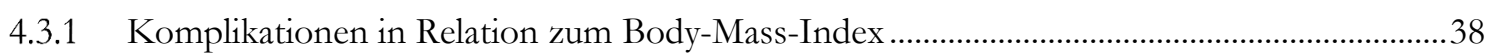

4.4 Schlussfolgerungen, Limitationen und Ausblick .........................................................................38

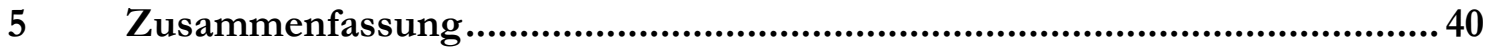

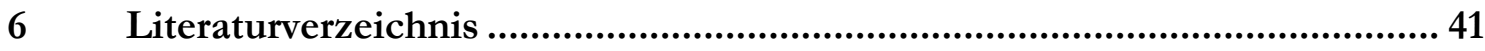




\section{Abbildungsverzeichnis}

Abbildung 1: Radiologischer Verlauf des Therapieerfolgs eines zehnjährigen Jungen mit spinaler Muskelatrophie während MAGEC®-Therapie.

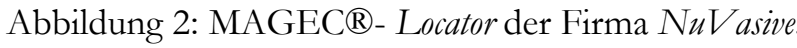

Abbildung 3: MAGEC ${ }^{\circledR}$ - external remote controller (ERC) der Firma NuV asive........................................14

Abbildung 4: Schematische Darstellung einer in der Frontalebene deformierten Wirbelsäule. ..........16

Abbildung 5: Bestimmung des Cobb-Winkels der Hauptkrümmung im a. p.-Röntgenbild. ...............17

Abbildung 6: Schemazeichnung zur Bestimmung der spinalen Länge (spinal lengtb).............................18

Abbildung 7: Messung der Distraktionslänge unter Berücksichtigung des

Vergrößerungsfaktors.

Abbildung 8: Korrelation der erreichten mit der spinalen Länge.

Abbildung 9: Verlängerung beider Implantate. Korrelation der erwarteten mit der erreichten Länge.

Abbildung 10: Anzahl der Patienten und Stabwechsel....................................................................27

Abbildung 11: Verlängerung konkaves Implantat. Korrelation der erwarteten mit der erreichten Länge...

Abbildung 12: Verlängerung konvexes Implantat. Korrelation der erwarteten mit der erreichten Länge.

Abbildung 13: Komplikationen in Relation zum BMI.

Abbildung 14: Veränderung des Cobb-Winkels in Grad präoperativ zu postoperativ für Kinder mit Komplikationen und ohne, getrennt betrachtet. .................................................................... 31

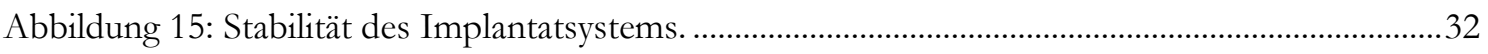

Abbildung 16: Varianz zwischen den beiden Untersuchern anhand des konvexen Stabauszuges.

\section{Tabellenverzeichnis}

Tabelle 1: Klinische Daten des Patientenkollektivs

Tabelle 2: Patientendaten 


\section{Abkürzungsverzeichnis}

$\begin{array}{ll}\text { a.p. } & \text { anterior-posterior } \\ \text { BMI } & \text { Body-Mass-Index } \\ \text { EOS } & \text { Early-Onset-Skoliose } \\ \text { ERC } & \text { external remote controller } \\ \text { GR } & \text { growing rods } \\ \text { MAGEC® } & \text { magnetic expansion control } \\ \text { MCGR } & \text { magnetically controlled growing rods } \\ \text { MRT } & \text { Magnetresonanztomographie } \\ \text { p.a. } & \text { posterior-anterior } \\ \text { SRS } & \text { Scoliosis } \text { Research Society } \\ \text { SMA } & \text { spinale Muskelatrophie } \\ \text { TIS } & \text { thoracic insufficiency syndrome } \\ \text { VEPTR } \mathbb{R} & \text { vertical expandable prosthetic titanium rib }\end{array}$




\section{Einleitung}

Wirbelsäulendeformitäten, die insbesondere im Wachstumsalter (Kindesalter) in Erscheinung treten, stellen häufig eine große Herausforderung in deren Versorgung dar und können unbehandelt mit schwerwiegenden gesundheitlichen Risiken für das Kind einhergehen (Akbarnia et al. 2005). Zu den Verformungen der Wirbelsäulen zählen Skoliosen, die definiert sind als seitliche, fixierte Verkrümmung eines oder mehrerer Wirbelsäulenabschnitte, welche als dreidimensionale Deformität zu verstehen sind (Bunnell 1988; Trobisch et al. 2010; Groenefeld und Hell 2013).

Häufig zeigen Wirbelsäulenveränderungen während der Wachstumsphase eine deutliche Zunahme ihrer Ausprägung, sodass es hierbei nicht nur zu einer starken kosmetischen und funktionellen Beeinträchtigung kommen kann, sondern auch die Ausbildung eines ThoraxInsuffizienz-Syndroms (thoracic insufficiency syndrome, TIS) droht (Campbell et al. 2003; Dannawi et al. 2013). Hierunter versteht man eine unzureichende Entwicklung des Brustkorbes, meist als Folge einer inadäquat behandelten Skoliose im Bereich der Brustwirbelsäule, die wiederum mit einem unzureichenden Lungenwachstum und somit eingeschränkter Lungenfunktion einhergehen kann (Scoliosis Research Society (SRS); Campbell et al. 2003). Daneben drohen auch kardiale Entwicklungsstörungen. Beides ist mit einer erhöhten Mortalität assoziiert (Sewell et al. 2016).

Umso wichtiger ist ein frühzeitiger Therapiebeginn der kindliche Wirbelsäulendeformitäten, um das Fortschreiten und damit einhergehende, oben angeführte gesundheitliche Folgen zu vermeiden (Jenks et al. 2014). Die Behandlung kann hierbei bei geringer Ausprägung zunächst konservativ (z. B. durch Korsette, Gipse oder Physiotherapie) erfolgen (Akbarnia et al. 2005).

Schnell fortschreitende Wirbelsäulendeformitäten, hierzu zählen insbesondere durch neurogene/muskuläre (neuromuskuläre) Erkrankungen oder Defekte der embryonalen Wirbelentwicklung (kongenitale) bedingte Skoliosen, lassen sich häufig durch konservative Verfahren nur unzureichend behandeln und in ihrem Progress nicht aufhalten, sodass schon bei jungen Kindern operative Wirbelsäulenaufrichtungen erfolgen müssen (Cheung et al. 2012; Poon et al. 2017; Hell et al. 2018).

In früheren Jahren erfolgten zur Behandlung von Wirbelsäulenverkrümmungen im Kindesalter sogenannte Versteifungsoperationen (Spondylodesen) der Wirbelsäule, um das weitere Fortschreiten $\mathrm{zu}$ verhindern. Folge dieser Operationen war jedoch durch das Unterbinden des Wachstums der kindlichen Wirbelsäule eine im Verhältnis zu kurze Wirbelsäule und ein zu kleiner Brustkorb mit Entwicklungsstörung der im Thorax befindlichen Organe (Karol 2011; Dannawi et al. 2013; Figueiredo et al. 2016). Zur Vermeidung der damit verbundenen Problematik wurden daher seit den 90er Jahren 
zunehmend wachstumsfreundliche (growth friendly-) Implantate wie growing rods (GR), vertical expandable prosthetic titanium rib (VEPTR) und als neuestes Produkt magnetically controlled growing rods (MCGR) eingesetzt (Hell et al. 2004; Campbell 2013; Poon et al. 2017; Thakar et al. 2018). Besonders die MCGR haben in den letzten Jahren zunehmend an Popularität gewonnen (Cheung et al. 2012; Akbarnia et al. 2013; Dannawi et al. 2013).

\subsection{Implantate für kindliche Wirbelsäulendeformitäten}

Ziel der operativen Versorgung von Wirbelsäulendeformitäten ist neben der Verbesserung von Thoraxgröße und -volumen (Campbell und Hell-Vocke 2003) in erster Linie die Korrektur der Deformität. Dabei sollte eine möglichst symmetrische Situation der Taillendreiecke, eine Balancierung des Rumpfes und ein Schultergradstand hergestellt werden. Die Deformität selber wird anhand des Cobb-Winkels klassifiziert. Unter dem Cobb-Winkel versteht man den seit 1948 zur Quantifizierung als Goldstandard verbreiteten Winkel, der zwischen den Geraden, die jeweils durch die Deckplatte des obersten und durch die Bodenplatte des untersten an der Wirbelsäulenverkrümmung beteiligten Wirbelkörpers gezogen wird, entsteht (Cobb 1948).

Eine operative Versorgung wird im Allgemeinen ab einem Cobb-Winkel von >40-50 Grad in Erwägung gezogen (Figueiredo et al. 2016), bzw. bei einer stark zunehmenden Deformität. Goldstandard in der heutigen operativen Versorgung kindlicher Wirbelsäulenverkrümmungen ist die Verwendung von wachstumsfreundlichen Systemen, die neben der Korrektur der Deformität das Wachstum der Wirbelsäule und somit auch das des Brustkorbes und der Lunge ermöglichen, um somit die Entstehung eines TIS zu vermeiden (Dannawi et al. 2013; Sewell et al. 2016). Das erste wachstumsfreundliche Implantat, die sogenannten growing rods, wurde bereits im Jahre 1962 von Paul Harrington entwickelt (Harrington 1962; Akbarnia et al. 2013).

Im Allgemeinen handelt es sich bei den Implantaten um uni- oder bilaterale, in der Länge veränderbare Metallstabsysteme, die proximal und distal der Wirbelsäulenverkrümmung mittels Schrauben, Haken und/oder Platten fixiert werden (Bekmez et al. 2017). Um das Wirbelsäulenwachstum weiterhin aufrechtzuerhalten, bleibt der dazwischenliegende Wirbelsäulenabschnitt unangetastet (Doany et al. 2017).

In den letzten Jahrzehnten wurden verschiedenartige wachstumsfreundliche Implantate entwickelt, die sich funktionell einteilen lassen in:

- Zug-basierte Systeme (growing rods, vertical expandable prosthetic titanium rib [VEPTR])

- Wachstumslenkende Systeme (Shilla, Luque-Trolley)

- Kompressionsbasierte Systeme (stapling, tethering) (Hosseini et al. 2016; Bekmez et al. 2017) 
Die genannten Verfahren sind Übergangsverfahren und dienen der vorübergehenden Therapie im Wachstum. In der Pubertät wird dann in den meisten Fällen eine Spondylodese (Wirbelsäulenversteifung) durchgeführt (Ridderbusch et al. 2013; La Rosa et al. 2017).

\subsubsection{Vertical expandable prosthetic titanium rib(VEPTR)}

Bei dem VEPTR-System (Firma Synthes Spine Co., Paoli, Pa.) handelt es sich um ein aus Titan gefertigtes Teleskop-Implantat, das in vertikaler Ausrichtung zur Wirbelsäule implantiert wird (Campbell und Hell-Vocke 2003; Hell et al. 2004). Eine häufige Indikation für die Anwendung des VEPTR-Verfahrens besteht bei Skoliosen mit fusionierten Rippen (Campbell 2013; Burnei et al. 2015). In Europa wurde das System 2002 erstmalig in der Universitätskinderklinik Basel, Schweiz, implantiert (Hell et al. 2004). Der Thorax soll während des Wachstums stabilisiert und aufgedehnt werden, um eine möglichst normale Entwicklung und Funktion der Lunge zu gewährleisten. Ferner soll die Verkrümmung der Wirbelsäule kontrolliert und korrigiert werden, ohne dabei das Wachstum der Wirbelsäule einzuschränken (Ramirez et al. 2009).

Beim VEPTR existieren drei verschiedene Implantatsysteme:

- „Rippe zu Rippe“-Implantat. Dieses führt durch Verankerung an den kranialen und kaudalen Rippen zu einer längsgerichteten Verstrebung des Brustkorbes.

- „Rippe zu Lendenwirbelsäule“-Implantat. Hier wird die Deformität mittels Fixierung an kranialen Rippen und der Lendenwirbelsäule aufgedehnt.

- „Rippe zu Becken“-Implantate werden zumeist parallel beidseits von kranialen Rippen zu den Beckenkämmen eingesetzt. Dieses Verfahren eignet sich besonders für nicht gehfähige Patienten und hat den Vorteil, dass die Wirbelsäule selber nicht tangiert wird.

\subsection{Nachteile bisheriger wachstumsfreundlicher Implantate}

Bei fast allen wachstumsfreundlichen Implantaten zur Wirbelsäulendeformitätenkorrektur im Kindesalter müssen wiederholte Operationen zur Verlängerung bzw. bei Komplikationen durchgeführt werden (Flynn et al. 2012). Üblicherweise erfolgen sowohl bei Verwendung der traditionellen growing rods (GR) als auch dem VEPTR-Verfahren Verlängerungsoperationen in einem Intervall von circa sechs Monaten bis zum Wachstumsabschluss und anschließender Spondylodese der Wirbelsäule (Dannawi et al. 2013). Bei der Anwendung von genannten Implantatsystemen besteht ein hohes Risiko für das Auftreten von Komplikationen aufgrund regelmäßiger Operationen (Kwan et al. 2017), der Komplexität der zu behandelnden Wirbelsäulendeformität sowie oft auch durch den schlechten gesundheitlichen Status betroffener Kinder (Campbell 2013; Thakar et al. 2018). Durch wiederholte invasive Verlängerungsprozeduren ist mit postoperativen Komplikationen wie Wundheilungsstörungen, Implantatinfektionen, -ausbrüchen und -dislokationen sowie Spontanfusionen und Ossifikationen der kindlichen Wirbelsäule häufig zu rechnen 
(Akbarnia und Emans 2010; Groenefeld und Hell 2013; Plaass et al. 2016; Wagner et al. 2018). Bess et al. beschrieben ein Risiko medizinischer Komplikationen von $24 \%$ pro Eingriff (Bess et al. 2010). Des Weiteren konnte durch Bess et al. 2010 in einer Nachuntersuchung von 140 Patienten (Gesamtzahl von 897 Operationen) ein individuelles Risiko von 58\%, eine Komplikation zu erleiden, nachgewiesen werden (Bess et al. 2010). Watanabe et al. beschrieben in einer Metaanalyse in Japan 2014 eine Komplikationsrate von 57\% bei Patienten, die mit growing rods behandelt worden (Watanabe et al. 2013). Ebenso muss die regelmäßige Belastung mit ionisierenden Strahlen bedacht werden (Mundis et al. 2016). Ferner führen wiederholte Krankenhausaufenthalte zu einer Beeinträchtigung der sozialen Entwicklung im Rahmen häufiger Fehlzeiten in der Schule (Cheung et al. 2012; Cheung et al. 2015). Auch Einschränkungen des Selbstwertgefühls können negative Auswirkungen auf soziale Beziehungen haben. In der betroffenen Population wird zudem ein erhöhtes Risiko für Depressionen beschrieben (Noonan et al. 1997). Häufig kommt es auch zu familiären Spannungen zum Beispiel durch Fehlzeiten bei der Arbeit und dadurch bedingten finanziellen Ausfällen (Bess et al. 2010; Figueiredo et al. 2016). Häufige Allgemeinanästhesien bergen zudem das Risiko von posttraumatischem Stress, Sprachstörungen und kognitiven Defiziten (Lebon et al. 2016), darüber hinaus erhöhen diese zunehmend die Mortalität sowie Morbidität der zumeist schwer vorerkrankten Kinder (Rolton et al. 2016; Thakar et al. 2018).

Limitierende Faktoren zum Einsatz dieser Verfahren sind weltweit gesehen die hohen Kosten der Therapie, eingeschränkter Zugang zur medizinischen Versorgung und Verfügbarkeit der Verfahren (Campbell et al. 2004).

\subsection{Extern zu kontrollierende magnetische Implantate}

Aufgrund der oben aufgeführten zahlreichen Nachteile bisheriger mitwachsender, wirbelsäulenaufrichtender Implantate erfolgte die Entwicklung sogenannter magnetically controlled growing rods (MCGR) (Cheung et al. 2012; Akbarnia et al. 2013; Hickey et al. 2014). Hierbei handelt es sich um ein ,neuartiges“ operatives Verfahren, das erstmals im Jahre 2009 durch Man-Chee Cheung (Hongkong) als Alternative zu den bisherigen mitwachsenden Stabsystemen in der Behandlung ausgeprägter Skoliosen eingesetzt wurde (Cheung et al. 2012). Die Idee magnetisch verlängerbarer growing rods wurde bereits 1998 durch Takaso et al. geäußert (Takaso et al. 1998).

Ausschlaggebende Motivation der Entwicklung war vor allem die Vermeidung regelmäßiger operativer (invasiver) Prozeduren und die weitere Verbesserung des möglichst naturgetreuen Wirbelsäulenwachstums durch häufigere Stabverlängerungen (Doany et al. 2017). Die Möglichkeit das Längenverhalten bei Implantaten über externe Magneten zu steuern, um Operationen zu vermeiden, findet bereits lange Zeit Anwendung bei Extremitäten-Prothesen (Wilkins und Soubeiran 2001). 
Das MAGEC@-System (magnetic expansion control) von der Firma NuV asive (San Diego, USA) besteht aus einem aus Titan gefertigten, sterilen Wachstumsstab und einer Fernsteuerung, dem sogenannten external remote controller (ERC) ((Dannawi et al. 2013). Es bietet im Vergleich zu den bisherigen mitwachsenden Wachstumsstäben den Vorteil der nicht-invasiven (nicht operativen) Stabverlängerungen durch das Auflegen eines extrakorporalen Magneten im ambulanten Rahmen (Cheung et al. 2012).

Indikation der Verwendung des MAGEC®-Konstruktes stellen Wirbelsäulendeformitäten dar, bei denen aufgrund eines bestehenden Restwachstums der Wirbelsäule eine Progression und somit Risiken pulmonale Komplikationen zu entwickeln, besteht (Cheung et al. 2015). Abhängig von der Flexibilität der Wirbelsäule können unterschiedliche Operationsverfahren und -techniken gewählt werden (Hell et al. 2018).

In Abhängigkeit vom Chirurgen werden ein bis zwei sterile Titanstäbe mit einem Durchmesser von 4,5, 5,5 oder 6,0 mm implantiert (Jenks et al. 2014; Rolton et al. 2016; Teoh et al. 2016). Bevorzugt eingesetzt werden wegen der besseren Stabilität zwei Stäbe, wobei im Einzelfall Einstabsysteme aufgrund der Konfiguration der Wirbelsäulendeformität oder bei sehr schmalem Habitus notwendig werden können (Cheung et al. 2015). Jeder MAGEC®-Stab besteht aus einem „aktiven“, der den internen Magneten beherbergt, und einem „nicht- aktiven“ Anteil (Rolton et al. 2016). Die Fixierung erfolgt entweder mit Schrauben und/oder Haken im kranialen und kaudalen Wirbelsäulenbereich (Bekmez et al. 2017) oder als neueres Verfahren parallel zur Wirbelsäule mit einer Rippen-zu-BeckenkammFixierung (Hell et al. 2018). Dieses Verfahren minimiert möglicherweise auch das beschriebene Risiko von Autofusion und heterotopen Ossifikationen (Groenefeld und Hell 2013).

Nach tierexperimentellen Versuchen mittels derer die Effektivität und Sicherheit des Systems bewiesen werden konnte (Akbarnia et al. 2012) erfolgte die Behandlung der ersten fünf Kinder mit Skoliose im Queen Mary Hospital in Hongkong im Zeitraum von November 2009 bis März 2013 durch Man-Chee Cheung und Kollegen (Cheung et al. 2012). Der Erfolg der Behandlung wurde anhand der vielversprechenden Ergebnisse nach 24 Monaten Beobachtungszeit zweier Patientinnen im Alter von fünf und zwölf Jahren demonstriert und im Jahr 2012 in „The Lancet" veröffentlicht (Cheung et al. 2012). Bei dem fünfjährigen Mädchen konnte eine Zunahme der Körpergröße von initial (kurz vor der Operation) 111,6 $\mathrm{cm}$ auf $125 \mathrm{~cm}$ verzeichnet werden. Die Armspannweite vergrößerte sich von 100,4 cm auf 109,5 cm. Die Messungen am zwölfjährigen Mädchen ergaben eine Zunahme der Körpergröße von $120 \mathrm{~cm}$ auf 142,7 cm sowie Zunahme der Armspannweite von $130 \mathrm{~cm}$ auf $143 \mathrm{~cm}$. Des Weiteren reduzierte sich der Cobb-Winkel der ersten Patientin deutlich von $74^{\circ}$ (präoperativ gemessen) auf $26^{\circ}$, bei der zweiten Patientin von $60^{\circ}$ auf $31^{\circ}$. Erfreulicherweise waren beide Patientinnen jederzeit schmerzfrei. Operationen waren in diesem Zeitraum keine notwendig. 
2013 veröffentlichten Akbarnia et al. vorläufige klinische Ergebnisse von 14 Patienten mit früh-aufgetretener Skoliose unterschiedlicher Ätiologie, der sogenannten Early-OnsetSkoliose (EOS), welche mit MAGEC® versorgt worden waren (Akbarnia et al. 2013). Die mittlere Anzahl der Distraktionen lag bei 4,9 in einem durchschnittlichen Abstand von 43 Tagen. Der präoperative Cobb-Winkel konnte von durchschnittlich $60^{\circ}$ auf $31^{\circ}$ zum letzten Kontrollpunkt reduziert werden. Hierbei ergaben sich keine Unterschiede zwischen der Versorgung mit einfachem oder doppeltem Stabsystem. Des Weiteren konnte eine Zunahme des Abstandes zwischen den Wirbelkörpern T1 und T12 bei den Patienten mit einem Stab von initial (durchschnittlich) $178 \mathrm{~mm}$ auf $204 \mathrm{~mm}$ zum letzten Kontrollzeitpunkt gemessen werden. Bei den Patienten mit doppeltem Stabsystem ergaben sich ähnliche Werte (Akbarnia et al. 2013).

Akbarnia et al. beschrieben ebenfalls eine annähernd vergleichbare Wirksamkeit in der Behandlung von traditionellen Wachstumsstäben zu extern magnetisch kontrollierbaren Systemen nach zwei Jahren (Akbarnia et al. 2014).

Sewell et al. wiesen nach, dass sich die Intensität postoperativer Schmerzen sowie die Reduktion der Aktivität und Teilhabe, im Vergleich zu konventionellen GR nicht wesentlich unterschied (Sewell et al. 2016).

\subsubsection{Verlängerungsprozedur}

Die Verlängerung der MAGEC®-Stäbe wird im ambulanten Rahmen am wachen Patienten durchgeführt. Eine Allgemeinanästhesie sowie Analgesie sind hierfür nicht notwendig (Dannawi et al. 2013).

Die Verlängerung oder Verkürzung des implantierten einfach- oder doppelten Stabsystems erfolgt durch Interaktion (Rotation) eines darin befindlichen Magneten mit einem externen Magneten, der auf dem Rücken des Patienten platziert wird (Bekmez et al. 2017). Über einen Schalter an der Fernsteuerung kann die Längenänderung des implantierten Stabsystems erfolgen. Das System kann dabei maximal auf bis zu $48 \mathrm{~mm}$ verlängert werden (Akbarnia et al. 2012; Rolton et al. 2016). Das Maß der Längenänderung erscheint auf dem Display (Akbarnia et al. 2012) und kann bis auf 0,1 mm genau gesteuert werden (Rolton et al. 2016). Die Dauer einer Verlängerungsprozedur beträgt dabei wenige Minuten (Cheung et al. 2012). Die Verlängerung erfolgt in einer Rate von $1 \mathrm{~mm} / 7$ Sekunden (Akbarnia et al. 2012).

Ein allgemeingültiges Konzept bezüglich des Behandlungsintervalls und das Maß der Längenänderung je Behandlungseinheit fehlen derzeit. In der Literatur beschriebene Intervalle werden durchschnittlich mit sechs Wochen bis sechs Monaten angegeben (Dannawi et al. 2013; Hickey et al. 2014; Lebon et al. 2016; Sewell et al. 2016; Ridderbusch et al. 2017). Geographische Faktoren (Verfügbarkeit spezieller Zentren) als beeinflussender Faktor der Häufigkeit von Verlängerungsprozeduren muss hierbei bedacht werden (Cheung et al. 2015). Im Allgemeinen gilt, dass durch kürzere Verlängerungsintervalle das Wirbelsäulenwachstum möglichst physiologisch, dem natürlichen Wachstum entsprechend, 
nachgeahmt werden soll und dass durch häufige und geringere Wirbelsäulenverlängerungen vermutlich Autofusionen vermieden werden (Cheung et al. 2017). Dem gegenüber steht jedoch die Gefahr von Anschlusskyphosen und Implantatversagen bei zu kurz gewählten Intervallen (Cheung et al. 2015). Die erforderliche Distraktionsstrecke wird in der Regel anhand der von DiMeglio entwickelten Wachstumskurven in Abhängigkeit vom Patientenalter berechnet (Dimeglio 2001).

Diese gliedern sich wie folgt (Dimeglio 2001):

- $\quad$ 0- 5 Jahre: jährliche Wachstumsrate (T1-L5) von 2,2 cm/ Jahr

- 5-10 Jahre: jährliche Wachstumsrate (T1-L5) von 1,1 cm/ Jahr

- >10 Jahre - Pubertät: jährliche Wachstumsrate (T1-L5) von 1,8 cm/ Jahr

Im Allgemeinen geht man hierbei von einer Wachstumsgeschwindigkeit von 1,5 mm pro Monat aus (Dannawi et al. 2013). Weitere Faktoren, die die Distraktionslänge beeinflussen, sind klinische Faktoren wie Schmerzen, Steifigkeit, bisherige Verlängerungsstrecke und Schwierigkeiten bei vorangegangenen Verlängerungsprozeduren. Gängige Verfahren zur Erfolgskontrolle jeder Distraktion sind eine Röntgendiagnostik oder Ultraschall (Lebon et al. 2016).

\subsubsection{Probleme bei Verlängerungsprozeduren}

Entgegen der Vorstellung der Reduktion hoher Komplikationsraten, die bei herkömmlichen wachstumsfreundlichen Implantaten beschrieben werden, findet man solche auch bei der Verwendung von magnetisch kontrollierten Wachstumsimplantaten. So beschrieben Lebon et al. eine Komplikationsrate von 57\% (Lebon et al. 2016), während Lorenz et al. eine geringere Komplikationsrate von 19\% aufzeigen konnten (Lorenz et al. 2017). Doany et al. beschrieben eine vergleichbare Anzahl ungeplanter Operationen infolge von Komplikationen bei konventionellen GR und MCGR (Doany et al. 2017).

In der Literatur beschriebene Komplikationen umfassen neben der Implantatlockerung, Implantatbruch, Wundheilungsstörungen mit oberflächlichen und tiefen Wundinfektionen, Anschlusskyphosierungen, die Ausbildung sekundärer Skoliosen, Lungenarterienembolie und pulmonale Insuffizienz, auch implantatspezifische (mechanische) Komplikationen (Akbarnia und Emans 2010; Bess et al. 2010; Watanabe et al. 2013; Plaass et al. 2016; Wagner et al. 2018). Hierunter zählen insbesondere die Blockierung des Stabsystems, teilweise mit Verkürzung des Stabes, das fehlende Ausfahren des Stabsystems sowie ein ab der vierten Sitzung beschriebenen Effektivitätsverlust (Lebon et al. 2016). Ferner wird in der Literatur eine ausbleibende Stabdistraktion durch fehlende komplette Rotation des internen Magneten beschrieben, erkennbar durch ein hörbares und tastbares „Klicken“ (sog. clunking phenomenon) (Cheung et al. 2017). Ebenfalls kann es zum Auftreten technischer Störungen kommen, beispielsweise bei fehlerhaftem Einbau des Stabsystems (Cheung et al. 2015). Des Weiteren wird von einer bestehenden Diskrepanz zwischen erwarteter und gemessener Verlängerung 
des Stabsystems (Streckenverlust) berichtet (Rolton et al. 2016). Insgesamt gibt es derzeit nur wenige Studien, die über den reellen Stabsauzug berichten.

Darüber hinaus wird in der Literatur vergleichend zu den konventionellen Stabsystemen von einer mit der Anzahl der Verlängerungsprozeduren zusammenhängenden Limitation der Wirksamkeit berichtet. Als ursächlich wird neben der zunehmenden Steifigkeit der Wirbelsäule durch Gewebsveränderungen, die Autofusion von Wirbelkörpern diskutiert (Sankar et al. 2011). Cheung et al. beschrieben einen Verlust der zu erwarteten Verlängerungsstrecke nach Erreichen der Hälfte der möglichen auszufahrenden Strecke (Cheung et al. 2017). Ferner wird die höhere Strahlenbelastung bei im Vergleich zu den herkömmlichen Systemen häufigeren radiologischen Kontrollen angeführt (Akbarnia et al. 2013).

Neben den allgemeinen Komplikationen, technischen/ mechanischen Schwierigkeiten, können patientenspezifische Faktoren die Wirksamkeit der Behandlung beeinflussen. Durch einen hohen Body-Mass-Index (BMI) und dadurch bedingten vergrößerten Abstand zwischen den Magneten ist ein Wirkverlust des Systems zu erwarten (Cheung et al. 2015). 


\subsection{Fragestellung der Dissertation}

In der vorliegenden Untersuchung wurden Kinder mit Wirbelsäulendeformität und nach Implantation von magnetisch zu kontrollierenden Implantaten retrospektiv untersucht. Alle ambulanten Verlängerungsprozeduren wurden evaluiert.

Dabei wurde folgende Hypothese untersucht:

Entspricht die auf dem externen Display (ERC) angezeigte Verlängerung in Millimetern $(\mathrm{mm})$ der auf dem Röntgenbild nachgemessenen Ausziehungen des Implantats?

Als sekundäre Fragestellungen wurden untersucht:

Können Risikofaktoren identifiziert werden, die zum Versagen der angestrebten Verlängerung führen?

Entspricht die ausgezogene Stablänge der veränderten Wirbelsäulenlänge?

Treten Komplikationen auf? 


\section{$2 \quad$ Material und Methoden}

\subsection{Ethikkommission}

Die vorliegende Untersuchung wurde im Rahmen der Ethikanträge „DOK_246_2015“ und „DOK_29_2013“ durch die Ethikkommission der medizinischen Fakultät der GeorgAugust-Universität Göttingen genehmigt.

\subsection{Studiendesign}

Die Studie wurde vom 01. Dezember 2011 bis 31. Dezember 2017 als retrospektive Kohortenstudie in der Kinderorthopädie unter der Leitung von Prof. Dr. med. AnnaKathrin Hell an der Klinik für Unfallchirurgie, Orthopädie und Plastische Chirurgie der Universitätsmedizin Göttingen durchgeführt. Seit genanntem Zeitraum werden in der Einrichtung Kinder mit Wirbelsäulendeformitäten mit extern zu steuernden magnetischen Implantaten zur Korrektur von kindlichen Wirbelsäulendeformitäten operativ versorgt. Alle Kinder erhielten wiederholte externe Verlängerungen, welche als erste Verlängerung fünf Monate nach Operation und dann in regelmäßigen Intervallen von drei Monaten stattfanden.

\subsubsection{Ein- und Ausschlusskriterien des Studienkollektivs}

In das Studienkollektiv wurden Kinder eingeschlossen, die den nachfolgenden Einschlusskriterien entsprachen:

- Kinder mit Wirbelsäulendeformitäten, welche mittels eines extern zu steuernden magnetischen Implantats (MAGEC $($ ), unabhängig von einer möglichen vorangegangenen wirbelsäulenfreundlichen Therapie (z. B. VEPTR), operativ in der Kinderorthopädie der UMG behandelt wurden

- Beobachtungszeitraum 01. Dezember 2011- 31. Dezember 2017

- Alter < 12 Jahre

- Erhalt von mindestens einer Verlängerungsprozedur

- Vorliegen mindestens eines Röntgenbildes der Wirbelsäule in zwei Ebenen (posterior-anterior und lateralem Strahlengang) präoperativ und nach der ersten Verlängerungsprozedur

Ausschlusskriterien:

- Kinder mit Wirbelsäulendeformitäten, welche andere operative oder konservative Verfahren erhielten

- Alter $>/=12$ Jahre

- Kinder, welche nach dem 31. Dezember 2017 ein von extern zu steuerndes magnetisches Implantat operativ eingesetzt bekamen 


\subsubsection{Endpunkt der Studie}

Der Endpunkt der Studie wurde anhand von vier Kriterien festgelegt. Diese umfassten den bis 31. Dezember 2017 definierten Beobachtungszeitraum, Ausbau des Implantats im Rahmen des Behandlungsabschlusses mit Wirbelsäulenversteifung sowie im Rahmen eines Therapieversagens oder dem Auftreten von Komplikationen, die das Fortführen der Therapie nicht weiter zuließen. Ferner das Versterben des Patienten oder Wechsel des Behandlungszentrums aufgrund eines räumlichen Umzugs.

\subsection{Patienten}

\subsubsection{Patientenkollektiv}

In der vorliegenden Studie wurden die Daten von 41 Kindern ( $=41)$ mit Wirbelsäulendeformität unterschiedlicher Ätiologie retrospektiv analysiert, die mit dem MAGEC@-Konstrukt (NuV asive, San Diego, USA; ehemals Firma Ellipse Technology, Inc., Irvine, CA, USA) operativ versorgt worden waren (Abbildung 1) und die Einschlusskriterien (Punkt 2.2.) erfüllten. 


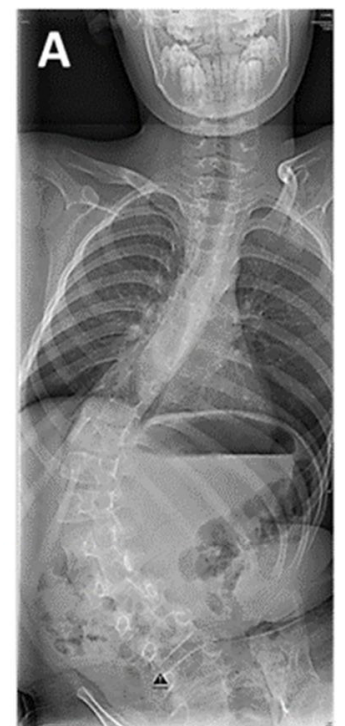

before
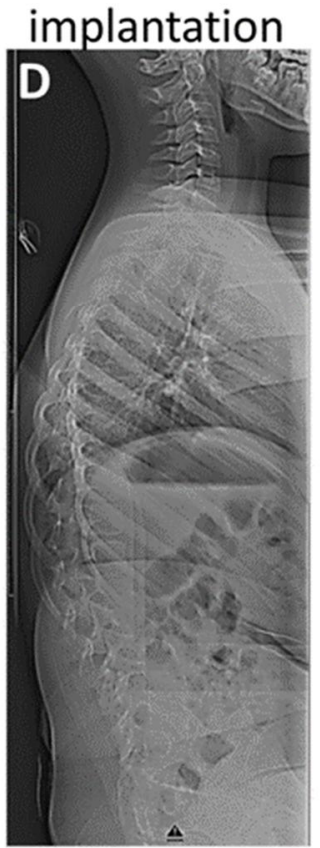

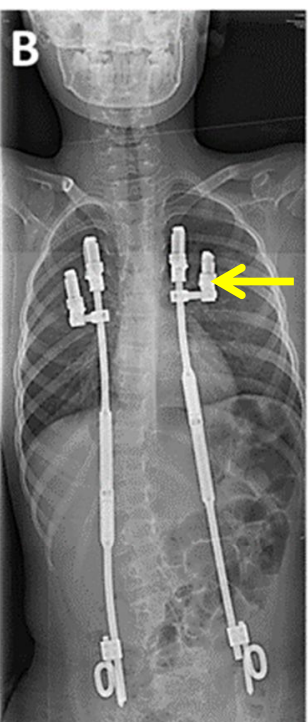

1 day after implantation

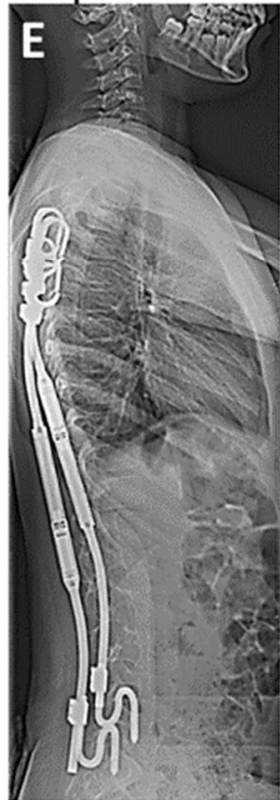

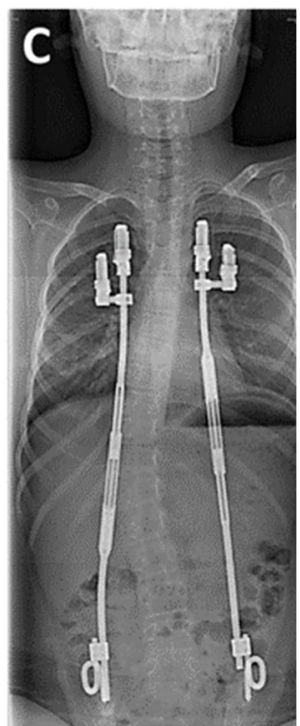

2 years of
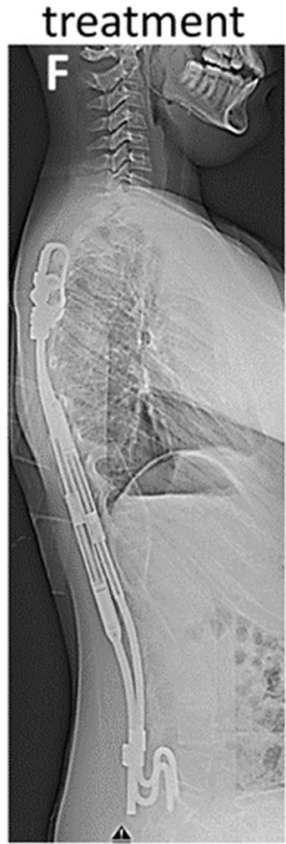

Abbildung 1: Radiologischer Verlauf des Therapieerfolgs eines zehnjährigen Jungen mit spinaler Muskelatrophie (Figure 1 aus Lorenz et al. 2018). Dargestellt sind die posteroanterior (A-C) und seitlichen Röntgenaufnahmen (D-F). Die Hauptkurve wurde von $81^{\circ}$ Kurvenwinkel (A) nach $27^{\circ}$ (B) nach Implantation der Vorrichtung korrigiert. Dieses Ergebnis konnte innerhalb des zweijährigen Follow-up (C) aufrechterhalten werden. Der gelbe Pfeil in dem Röntgenbild B weist auf den lateralen Ausleger (siehe unten). Patienteneinwilligung vorhanden. 


\subsubsection{Behandlungsablauf}

Nach sorgfältiger Indikationsstellung und Aufklärung der Patienten erfolgte die operative Implantation eines uni- oder bilateralen MAGEC®-Stabsystems in Vollnarkose. In der Mehrzahl wurden bilaterale Rippe-zu-Beckenkamm-Verankerungen implantiert, welche teilweise im Rippenbereich durch einen jeweiligen lateralen Ausleger eine zusätzliche Stabilität erhielten (siehe gelber Pfeil in Abbildung 1).

Nach abgeschlossener Wundheilung und Mobilisation wurden die Kinder nach Hause entlassen. Eine erste ambulante Verlängerung erfolgte fünf Monate nach der Operation. Danach wurden Intervalle von drei Monaten eingehalten.

Für die ambulante Verlängerung wurden die Kinder in eine Bauch- oder Seitenlage bzw. eine sitzende Position (Abbildung 2 und 3) gebracht, um den zu distrahierenden Anteil des Stabsystems zunächst durch Ertasten und unter Hinzunahme des MAGEC®-Locator zu identifizieren.

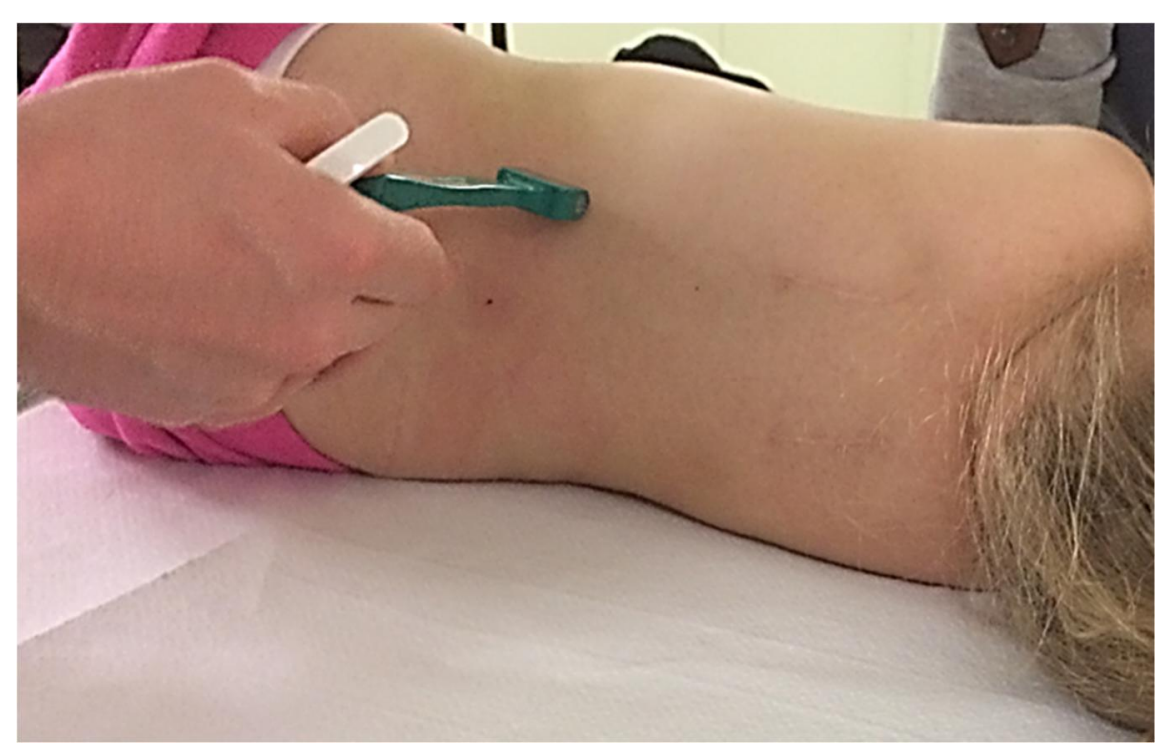

Abbildung 2: MAGEC®-Locator der Firma NuV asive. Externer Magnet, der das Aufsuchen des im Titanstab integrierten Magneten erleichtert. Patienteneinwilligung vorhanden.

Anschließend erfolgte die Distraktion extrakorporal durch Auflegen des external remote controller (ERC) (Abbildung 3). Auf dem Display wurde die Verlängerungsstrecke in Millimeter $(\mathrm{mm})$ angezeigt. Als Distraktionsstrecke wurde dabei regulär $5 \mathrm{~mm}$ pro Implantat und Behandlungseinheit gewählt. In Einzelfällen musste hiervon zum Beispiel bei Auftreten von Schmerzen, Komplikationen oder in Abhängigkeit von der Körpergröße geplant individuell abgewichen werden. 


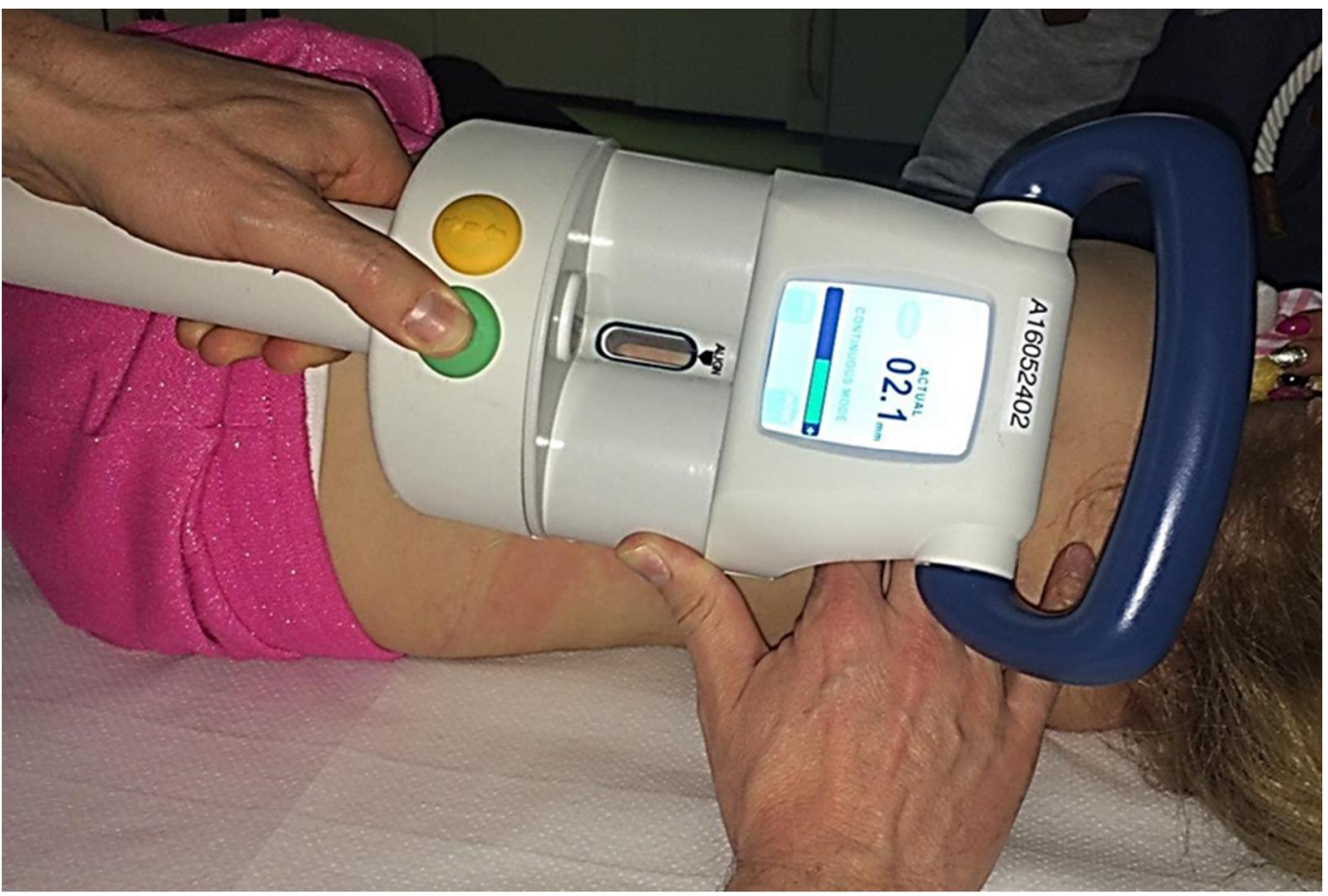

Abbildung 3: MAGEC®-external remote controller (ERC) der Firma NuV asive. Extrakorporaler Magnet mit dem das Stabsystem auf die gewünschte Länge distrahiert wird. Die veränderte Länge erscheint dabei auf dem Display. Patienteneinwilligung vorhanden.

Bis zum Jahr 2014 erfolgten vor und nach der Distraktion jeweils Röntgenaufnahmen in zwei Ebenen (posteroanteriorer und lateraler Strahlengang), um die korrekte Lage des Stabsystems sowie den gewünschten Verlängerungseffekt bildmorphologisch zu identifizieren und zu dokumentieren. In den nachfolgenden Jahren wurden zur Strahlenreduktion bei den Kindern jeweils Röntgenbilder ausschließlich nach jeder Verlängerungsprozedur angefertigt.

\subsection{Datenerhebung}

\subsubsection{Klinische Daten}

Hinsichtlich der Fragestellungen relevante Patientendaten wurden hinsichtlich folgender Parameter erhoben:

- Geburtsdatum

- Geschlecht

- Haupt- und Nebendiagnosen

- Gehfähigkeit

- Alter bei Erstimplantation des MAGEC®-Systems

- operative Vorbehandlungen (z. B. VEPTR)

Zur Berechnung des Body-Mass-Index wurden die Körpergröße und das Körpergewicht aus den elektronischen Arztbriefen bzw. Operationsberichten der jeweiligen 
Verlängerungsprozedur mithilfe der Kommunikationsplattform ixserv® (Version 4.25, ixmid Software Technologie GmbH, Köln) dokumentiert. Zusätzlich wurde die Anzahl und Art möglicher Komplikationen während des Therapieverlaufs notiert.

\subsubsection{Body-Mass-Index (BMI)}

Der Body-Mass-Index ist ein einfaches Messverfahren zur Ermittlung des Ernährungszustandes eines Menschen mithilfe dessen indirekte Rückschlüsse auf den Körperfettanteil gezogen werden können (Kromeyer-Hauschild et al. 2001). In die Berechnung einfließende Faktoren sind das Körpergewicht in Kilogramm [kg] und die Körpergröße in Metern [m]. Daraus ergibt sich die Einheit kg/ $\mathrm{m}^{2}$.

Der Body-Mass-Index wird dann anhand folgender Formel errechnet:

Körpergewicht [kg]: Körpergröße [m] $]^{2}$ (Dietz und Bellizzi 1999).

Die alleinige Anwendung des BMI zur Abschätzung des Ernährungszustandes bei Kindern eignet sich aufgrund alters- und geschlechtsabhängiger physiologischer Schwankungen des Anteils von Körperfett zu Knochen- und Muskelmasse nicht, sodass hierbei BMIReferenzkurven (Perzentilen) Verwendung finden (Kromeyer-Hauschild et al. 2001).

In Deutschland gültige Definitionen für Übergewicht und Adipositas richten sich nach der Arbeitsgemeinschaft „Adipositas im Kindes- und Jugendalter“ (AGA) wie folgt in:

- Übergewicht: BMI-Perzentile $>90-97$

- Adipositas: $>97-99,5$

- Extreme Adipositas: >99,5 (Adipositas im Kindes- und Jugendalter)

Analog dazu ergibt sich die Definition einer Unterernährung:

- Untergewicht: BMI-Perzentile $<10$

- Starkes Untergewicht: BMI-Perzentile $<3$

Der Bereich zwischen der zehnten und 90. Perzentile sind einem Normalgewicht zugeordnet. In das Studienprotokoll ist der BMI zum Zeitpunkt des Auftretens von Komplikationen berücksichtigt worden. Die Bestimmung dessen mit der gleichzeitigen Ermittlung der jeweiligen Perzentile erfolgte elektronisch mittels eines BMI-Rechners auf der Internetseite des Universitätsklinikums für Kinder- und Jugendmedizin Tübingens (Tübingen BMIRechner). Dafür benötigte Daten wie Geburtsdatum, Datum des berücksichtigten Körpergewichts und der Körpergröße wurden der jeweiligen Akte entnommen.

\subsubsection{Radiologische Datenerfassung}

Von allen Patienten, die aufgrund einer Wirbelsäulendeformität mit dem MAGEC®Konstrukt versorgt wurden oder sich gegenwärtig noch in Behandlung befinden, wurde vor Erstimplantation ein Röntgenbild der Wirbelsäule im Sitzen oder Stehen im a. p. und lateralen Strahlengang angefertigt. Zudem erfolgten radiologische Kontrollen vor und nach 
jeder Verlängerungsprozedur. Die für die Studie relevanten Messungen erfolgten digital im Verarbeitungsprogramm Centricity Enterprise Web V3.0 (GE Healthcare).

Insgesamt wurden 802 Röntgenbilder untersucht.

Um die Messgenauigkeit zu erhöhen, wurden die Röntgenbilder von zwei Untersuchern unabhängig voneinander ausgewertet. Sollten hierbei Differenzen entstanden seien, ist der Mittelwert aus beiden Messergebnissen ermittelt und in der Studie berücksichtigt worden.

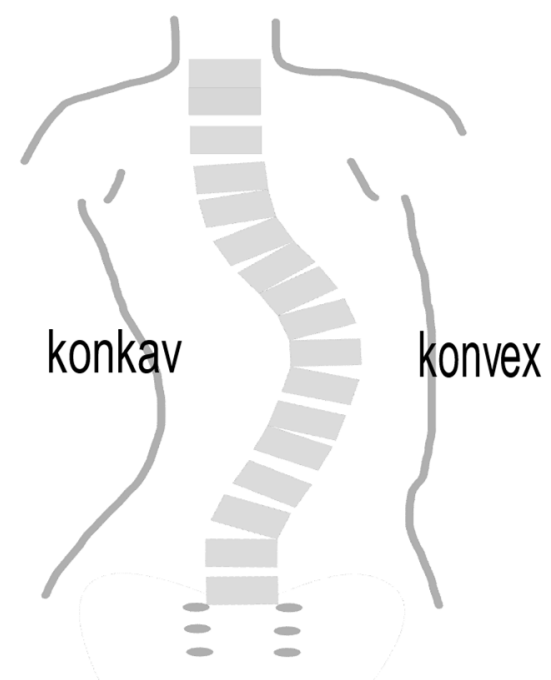

Abbildung 4: Schematische Darstellung einer in der Frontalebene deformierten Wirbelsäule von dorsal mit Angabe der Lagebeziehung im Körper durch die Begriffe „konkav“/ „konvex“.

\subsubsection{Skoliosekrümmungswinkel}

Im a. p. Röntgenbild wurde der Winkel der Hauptkrümmung anhand der Cobb-Methode (Cobb 1948) bestimmt. Dazu wurden jeweils der kraniale und kaudale Endwirbel der Hauptkrümmung bestimmt, welche sich dadurch definieren, dass sie am meisten in Richtung der Krümmung geneigt sind und somit am stärksten von der Horizontalen abweichen. Danach wurden an die Deck- bzw. Grundplatten der festgelegten Endwirbel Geraden angelegt. Der Schnittwinkel der Geraden ergibt den Cobb-Winkel (Abbildung 5). 


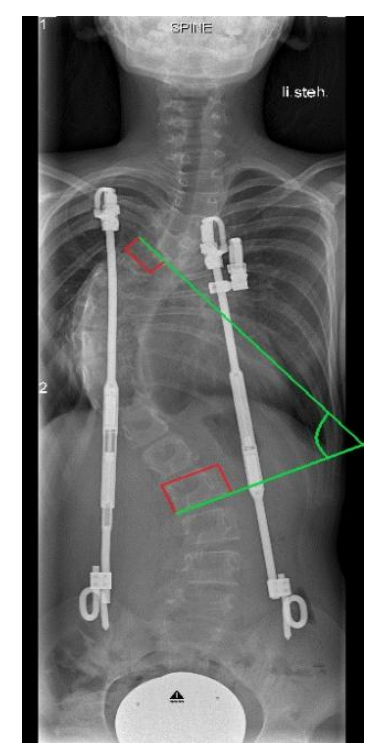

Abbildung 5: Bestimmung des Cobb-Winkels der Hauptkrümmung im a. p.-Röntgenbild. Der Winkel wird durch zwei Geraden definiert, die parallel zur Deckplatte des oberen Endwirbels und parallel zur Bodenplatte des unteren Endwirbels liegen. Patienteneinwilligung vorhanden.

\subsubsection{Wirbelsäulenlänge (spinal length / spinale Länge)}

Ziel der Behandlung des MAGEC®-Stabsystems ist, unter der Ermöglichung des physiologischen Wirbelsäulenwachstums die vorliegende Wirbelsäulendeformität auszugleichen. Um den Behandlungserfolg zu objektivieren, erfolgte die Ermittlung des Wirbelsäulenwachstums anhand der spinalen Länge (spinal length). Für genannte Messgröße wurde sich aufgrund der häufig fehlenden Kopfkontrolle der Patienten entschieden.

Die spinale Länge ist definiert als Vertikale zwischen Mittelpunkt des Wirbelkörpers auf Höhe dessen die kraniale Verankerung des Stabsystems (Rippenhaken) liegt zum Promontorium ossis sacri, der den Übergang der Lendenwirbelsäule zum Steißbein darstellt. Die Vertikale muss dabei in einem rechten Winkel zu einer Waagerechten stehen, die durch den unteren Messpunkt gelegt wird (Abbildung 6). 


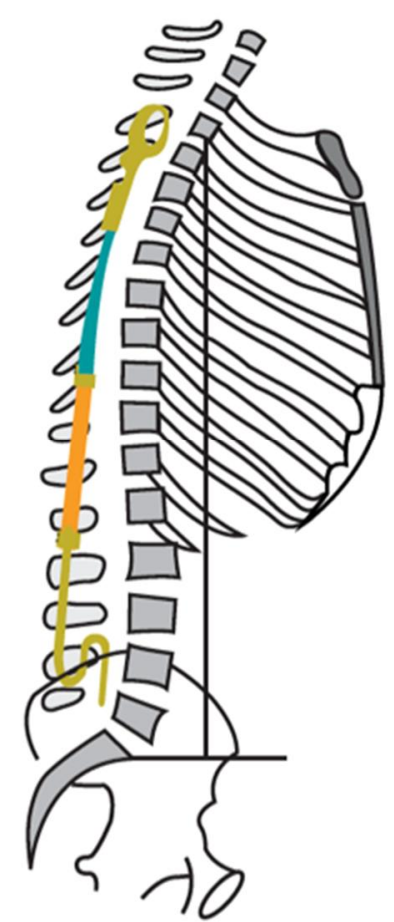

Abbildung 6: Schemazeichnung zur Bestimmung der spinalen Länge (spinal length). Die Abbildung zeigt schematisch eine Wirbelsäule im sagittalen Profil. Eingezeichnet ist ein MAGEG®-Stabsystem. Die Longitudinale gibt dabei die spinale Länge zwischen dem kranial gelegenen Rippenhaken und der Vertikalen von der Vorderkante des Promontoriums an. (Eigene Skizze, orientierend erstellt an Skizze von Dr. med. H. Lorenz, Arbeitsgruppe Kinderorthopädie, UMG, 2015).

\subsubsection{Auszug der Implantate}

In einer achtfachen Vergrößerung des a. p. Röntgenbildes wurde die Auszuglänge pro Behandlungseinheit, jeweils seitengetrennt für das Implantat der konkaven und der konvexen Seite an der Gehäuseeinheit des MAGEC®-Stabes ermittelt (Abbildung 7). Das bilaterale Stabsystem wird gegenläufig implantiert. Somit können die Implantate einfacher einzeln mit dem ERC gesteuert und hierdurch das Risiko eines unkontrollierten Ausfahrens beider Implantate minimiert werden. Um einen Vergrößerungs- oder Verkleinerungsfehler ausschließen zu können, wurde zusätzlich zur Auszugslänge auf jedem Röntgenbild auch der Durchmesser des MAGEC®-Stabes ausgemessen. Dieser beträgt laut Hersteller ( $N u V$ asive, ehemals Ellipse Technologies, Inc., Irvine, California, USA) 9,02 mm. Wurde beim Ausmessen dieses Durchmessers ein anderer Wert ermittelt, so wurde der gemessene Auszugswert mittels Dreisatz entsprechend dem Vergrößerungsfaktor angepasst.

Die erwartete Distraktionslänge gab den Wert wieder, der als gewünschte Verlängerung in den ERC eingegeben wurde. Dieser betrug regulär $5 \mathrm{~mm}$ und wurde nur in Einzelfällen individuell angepasst. 
Die Abweichung der erwarteten und erreichten Distraktionslänge wurde im Diagramm dargestellt.

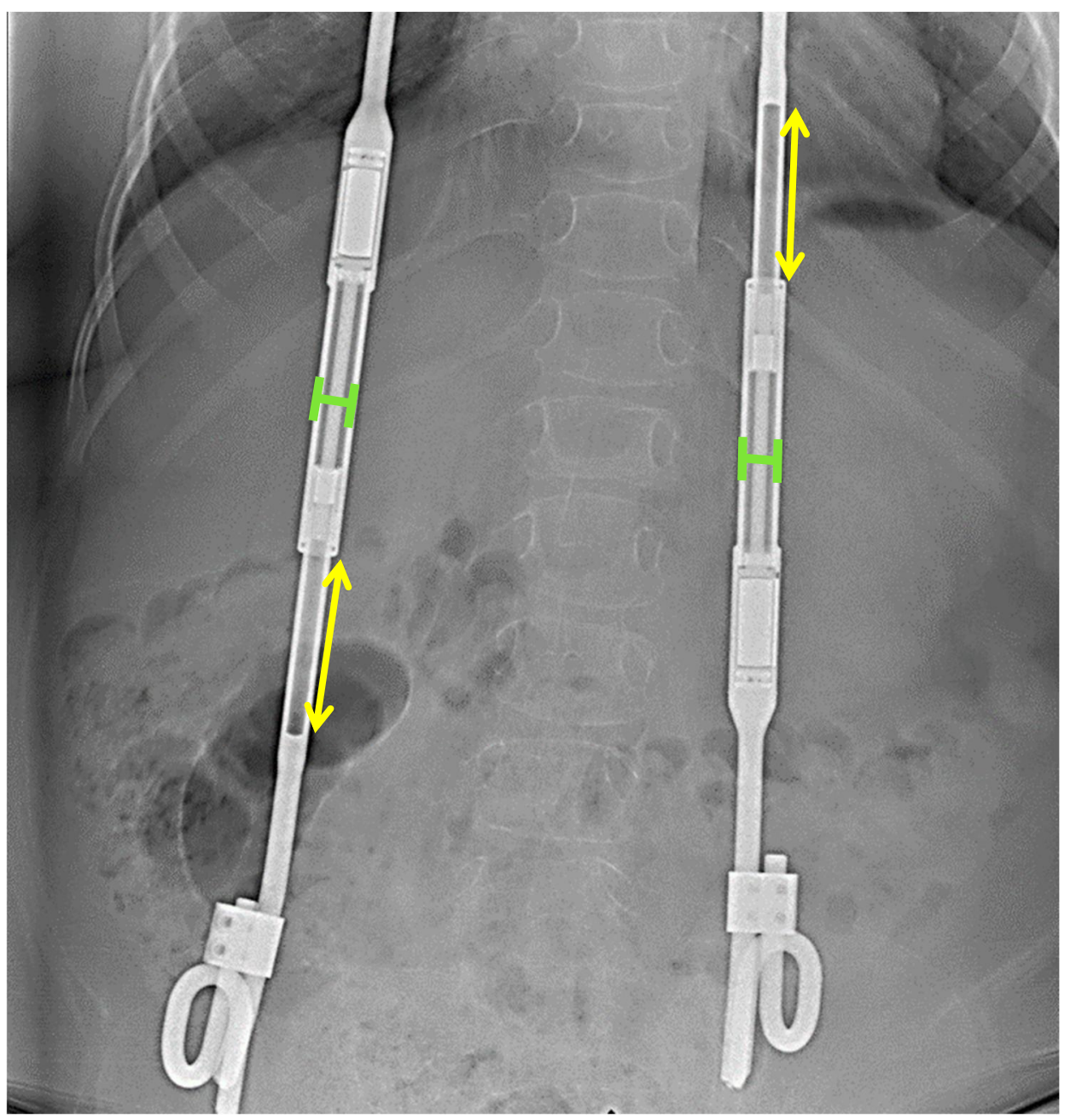

Abbildung 7: Die Messung der Distraktionslänge (gelb) ist in Abbildung 7 dargestellt (Figure 1 aus Lorenz et al. 2018). Zur Korrektur des Vergrößerungsfaktors wurde der Implantatdurchmesser (grün) in jeder Röntgenaufnahme ermittelt und mit dem tatsächlichen Wert verglichen. Patienteneinwilligung vorhanden. 


\subsection{Statistische Analyse}

Die erhobenen Daten wurden in eine Tabelle des Programms Access (Microsoft Corporation, Redmond, USA) eingefügt, die alle je erhobenen Messwerte aus der Abteilung der Kinderorthopädie umfasst. Zur statistischen Auswertung erfolgte die Übertragung der für diese Arbeit relevanten Daten in pseudonymisierter Form in eine Excel Tabelle (Microsoft Corporation, Redmond, USA). Die statistische Analyse und die Darstellung der Ergebnisse mittels Balken- und Liniendiagrammen erfolgten ebenfalls mit dem Programm Excel.

\subsubsection{Inter-Observer-Fehler}

Um die Ergebnisse zu validieren, wurden alle Messungen von zwei unabhängigen Untersuchern durchgeführt. Die Werte wurden gemittelt. Der daraus gewonnene Mittelwert wurde für die weitere statistische Analyse verwendet.

Die Messabweichung zwischen den beiden Untersuchern wurde als mittlerer Messfehler bestimmt und beispielhaft für die Auszugslänge dargestellt (siehe Abbildung 16). 


\section{Ergebnisse}

\subsection{Patientenkollektiv}

Von den in der Studie berücksichtigten Patienten ( $\mathrm{n}=41$ ) waren 21 weiblichen (entspricht $51,2 \%$ ) und 20 (entspricht 48,8\%) männlichen Geschlechts. Daraus ergibt sich ein Geschlechterverhältnis (Jungen : Mädchen) von 0,95. Die Ätiologie der Skoliose war neuromuskulär $(n=30)$, kongenital $(n=6)$ und syndromal $(n=4)$ sowie idiopathisch $(n=1)$.

Als Grunderkrankung der neuromuskulären und syndromalen Skoliosen lagen in den meisten Fälle $(n=27)$ spinale Muskelathrophien verschiedenen Typs vor, gefolgt von der Zerebralparese ( $\mathrm{n}=2$ ), ein Fall von intraspinaler Neoplasie und vier Syndromen (siehe Tabelle $1)$.

Sieben der 41 Patienten (17\%) waren gehfähig. 34 Kinder waren im Rollstuhl mobilisiert.

Das Durchschnittsalter bei Erstimplantation des MAGEC®-Stabsystems in Jahren lag bei 7,5 (2,9-11,6). Ein bilaterales Stabsystem erhielten dabei insgesamt 38 Patienten (92,7\%), mit einem unilateralen wurden drei Patienten $(7,3 \%)$ versorgt. Die Verankerung erfolgte mittels Rippenring und Beckenhaken. Bei 38 Patienten lag ein Rippen-zuLendenwirbelsäulenkonstrukt vor. Von den 41 berücksichtigten Patienten waren zehn Patienten $(24,4 \%)$ vor der MAGEC®-Implantation über eine mittlere Dauer von 30 Monaten mit dem VEPTR-System vorbehandelt worden. Im Mittel erfolgten neun magnetische Verlängerungsprozeduren pro Patient (1-17) in einem zeitlichen Intervall von drei Monaten. Der durchschnittliche Behandlungszeitraum betrug 32,6 Monaten. Die kürzeste Behandlungsdauer lag hierbei bei fünf Monaten, die längste bei insgesamt 57,3 Monaten. Während des Behandlungszeitraumes musste das MAGEC®-Stabsystem in insgesamt 22 Fällen getauscht werden, wovon 18 Fälle planmäßig wegen eines kompletten Ausfahrens des Implantates erfolgten. In vier Fällen wurde das Implantat wegen Implantatversagens (z. B. Implantatbruch) ausgetauscht. 
Tabelle 1: Klinische Daten des Patientenkollektivs.

\begin{tabular}{lc}
\hline Variable & Wert \\
\hline Anzahl der Patienten & 41 \\
Weiblich & 21 \\
Männlich & 20 \\
\hline MAGEC®-Therapie & $7,5(2,9-11,6)$ \\
Durchschnittsalter bei Erstimplantation & $9,0(1-17)$ \\
Anzahl der Verlängerungen & $32,6(5,0-57,3)$ \\
Dauer Behandlung in Monaten & 10 \\
VEPTR®- Vorbehandlung & 1 \\
\hline Skolioseätiologie & 1 \\
Idiopathische Skoliose & 30 \\
Neuromuskuläre Skoliose & 10 \\
Kongenitale/ syndromale Skoliose & 27 \\
\hline Grunderkrankung & 2 \\
SMA & 1 \\
Zerebralparese & 1 \\
Intraspinale Neoplasie & \\
Partielle Trisomie 9 & 1 \\
Goldenhar-Syndrom & \\
\hline Prader-Willi-Syndrom & 1 \\
\hline Marfansyndrom & \\
\hline
\end{tabular}


Tabelle 2: Patientendaten. Legende: IS= Idiopathische Skoliose; NMS= Neuromuskuläre Skoliose; KS= kongenitale/syndromale Skoliose

\begin{tabular}{|c|c|c|c|c|c|}
\hline 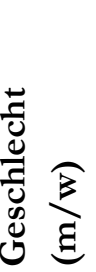 & 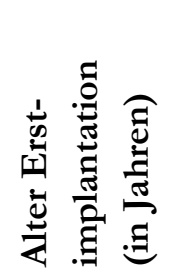 & 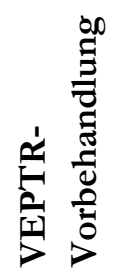 & 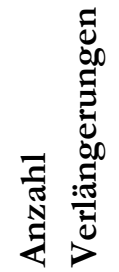 & 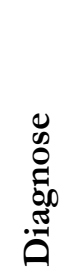 & 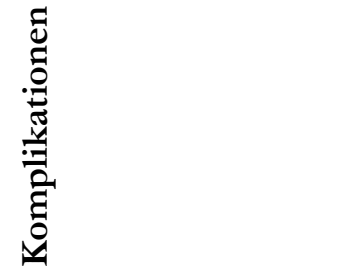 \\
\hline W & 7,8 & Nein & 3 & IS & Implantatversagen \\
\hline W & 3,9 & $\mathrm{Ja}$ & 7 & KS & Implantatversagen $(2 \mathrm{x})$ \\
\hline W & 4,7 & $\mathrm{Ja}$ & 2 & KS & \\
\hline W & 5,9 & $\mathrm{Ja}$ & 12 & KS & $\begin{array}{l}\text { Infektion, } \\
\text { Implantatversagen }\end{array}$ \\
\hline
\end{tabular}

\begin{tabular}{|c|c|c|c|c|c|c|}
\hline 5 & W & 6,5 & Nein & 3 & KS & \\
\hline 6 & W & 11,6 & $\mathrm{Ja}$ & 8 & KS & \\
\hline 7 & M & 10,9 & $\mathrm{Ja}$ & 5 & KS & Implantatversagen \\
\hline 8 & M & 5,0 & Nein & 9 & KS & \\
\hline 9 & M & 5,9 & Nein & 7 & KS & Implantatversagen \\
\hline 10 & M & 2,9 & Nein & 11 & KS & Implantatversagen \\
\hline 11 & M & 10,8 & Nein & 17 & $\mathrm{KS}$ & \\
\hline 12 & W & 4,7 & $\mathrm{Ja}$ & 3 & NMS & \\
\hline 13 & W & 5,2 & Nein & 11 & NMS & \\
\hline 14 & W & 5,5 & Nein & 9 & NMS & \\
\hline 15 & W & 6,2 & Nein & 14 & NMS & Implantatversagen \\
\hline 16 & $\mathrm{~W}$ & 6,3 & nein & 6 & NMS & \\
\hline 17 & W & 7,0 & Nein & 12 & NMS & Dekubitus \\
\hline 18 & W & 7,2 & Nein & 10 & NMS & \\
\hline 19 & W & 7,4 & Nein & 13 & NMS & Implantatversagen \\
\hline 20 & W & 7,6 & Nein & 10 & NMS & \\
\hline 21 & W & 8,1 & Nein & 9 & NMS & \\
\hline 22 & M & 8,2 & $\mathrm{Ja}$ & 16 & NMS & Implantatversagen \\
\hline 23 & $\mathrm{~W}$ & 8,3 & Nein & 15 & NMS & Dekubitus, fehlendes Ausfahren \\
\hline 24 & W & 8,7 & Nein & 11 & NMS & Implantatversagen \\
\hline 25 & $\mathrm{~W}$ & 9,6 & Nein & 5 & NMS & \\
\hline 26 & $\mathrm{~W}$ & 10,4 & $\mathrm{Ja}$ & 9 & NMS & fehlendes Ausfahren \\
\hline
\end{tabular}




\begin{tabular}{|c|c|c|c|c|c|c|}
\hline & 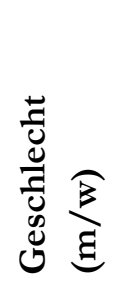 & 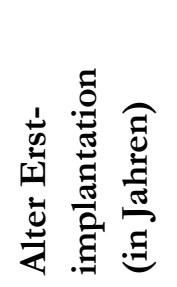 & 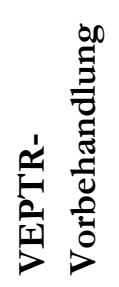 & 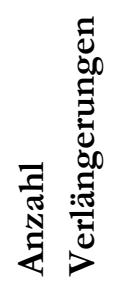 & 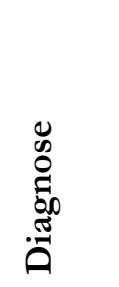 & 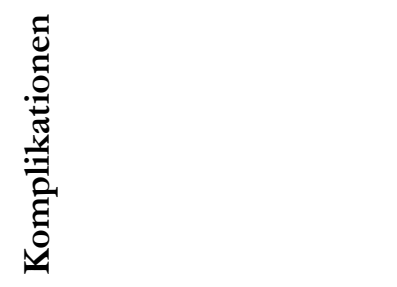 \\
\hline 27 & $\mathrm{M}$ & 5,1 & Nein & 13 & NMS & fehlendes Ausfahren \\
\hline 28 & M & 5,5 & Nein & 4 & NMS & beidseitiges Ausfahren \\
\hline 29 & M & 5,7 & Nein & 1 & NMS & \\
\hline 30 & M & 6,2 & Nein & 9 & NMS & \\
\hline 31 & M & 6,7 & Nein & 2 & NMS & \\
\hline 32 & M & 7,8 & $\mathrm{Ja}$ & 13 & NMS & Infektion \\
\hline 33 & M & 8,2 & Nein & 10 & NMS & Implantatversagen \\
\hline 34 & M & 8,2 & Nein & 14 & NMS & Infektion, Dekubitus \\
\hline 35 & M & 8,6 & Nein & 16 & NMS & $\begin{array}{l}\text { fehlendes Ausfahren (6x), } \\
\text { Implantatversagen }\end{array}$ \\
\hline 36 & M & 9,2 & Nein & 15 & NMS & \\
\hline 37 & M & 9,2 & Nein & 11 & NMS & \\
\hline 38 & M & 9,4 & Nein & 13 & NMS & \\
\hline 39 & M & 10,2 & Nein & 4 & NMS & \\
\hline 40 & M & 10,9 & Nein & 6 & NMS & \\
\hline 41 & M & 11,5 & $\mathrm{Ja}$ & 11 & NMS & $\begin{array}{l}\text { fehlendes Ausfahren }(4 \mathrm{x}) \text {, } \\
\text { Implantatversagen }\end{array}$ \\
\hline
\end{tabular}

\subsection{Radiologische Wirbelsäulendeformitätenparameter}

Zur Objektivierung des Behandlungserfolges wurde die erreichte Länge des MAGEC®Stabes mit der Wirbelsäulenlänge korreliert. Hierzu wurde die spinale Länge, wie sie in Kapitel 2.4.3.2 erläutert wurde, als radiologischer Parameter herangezogen.

In Abbildung 8 ist daher die erreichte Länge in $\mathrm{mm}$ (rot) für beide Stabsysteme zusammengefasst sowie die spinale Länge in mm (blau) über die Anzahl der Verlängerungen aufgeführt.

Hierbei lässt sich eine deutliche Abhängigkeit der spinalen Länge von der erreichten Implantatlänge erkennen. Somit lässt sich eindeutig zeigen, dass das MAGEC®-System direkte Auswirkungen auf die Spinallänge des Patienten hat und damit schlussfolgernd auch 
den erwünschten positiven Einfluss auf die Körpergröße (bzw. Größe des Oberkörpers) nimmt. Die kurzfristige, zum Zeitpunkt der zehnten Verlängerung, aufgetretene plötzliche Zunahme der spinalen Länge gegenüber der erreichten Implantatlänge ist auf den Implantataustausch bei 14 Patienten zwischen der neunten und zwölften Verlängerung zurückzuführen.

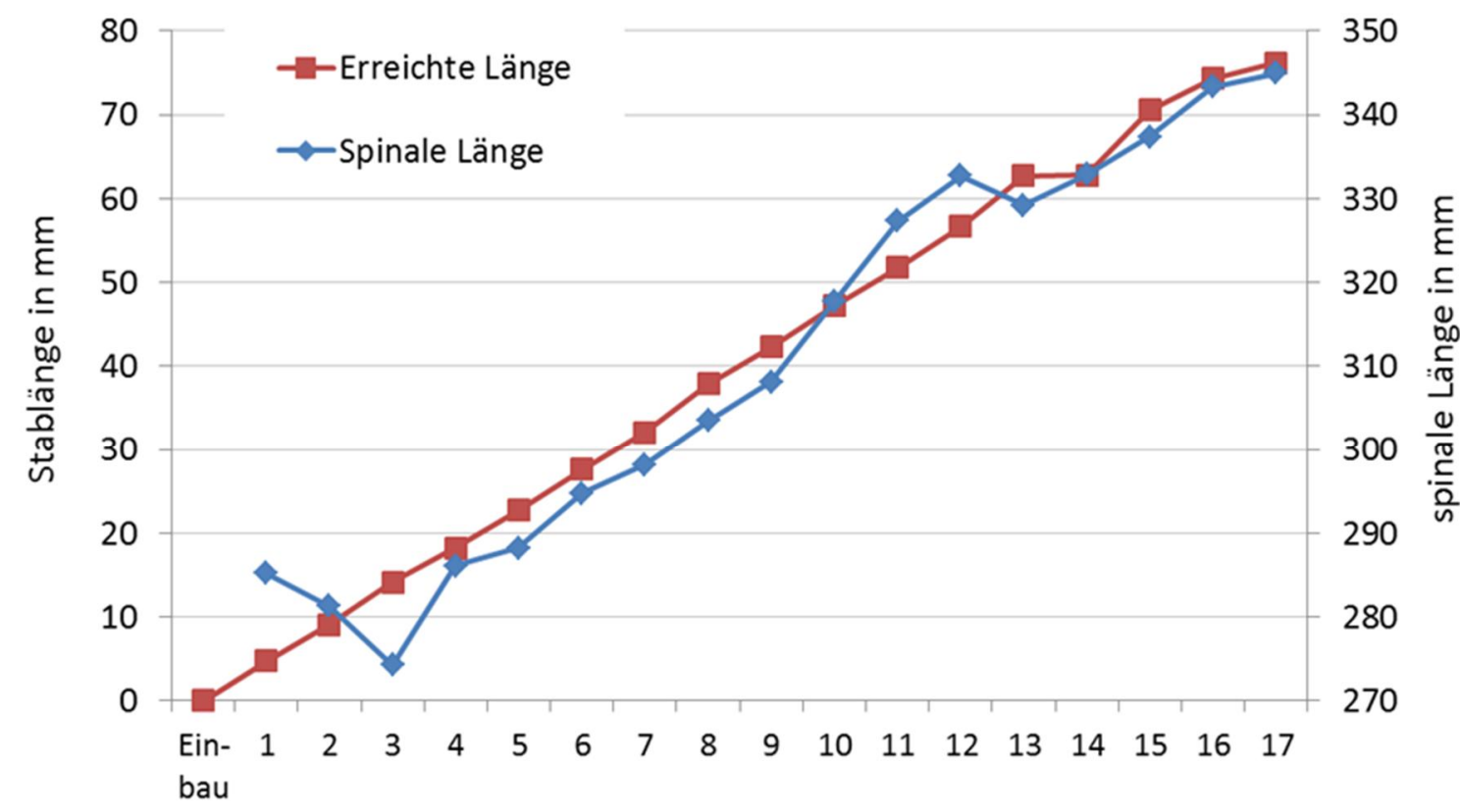

Anzahl der Verlängerungen

Abbildung 8: Korrelation der erreichten mit der spinalen Länge. Korrelation zwischen der erreichten Distraktionslänge und der Länge der Wirbelsäule in Millimetern. Zwischen den Verlängerungen 9 und 12 erhielten 14 Kinder einen chirurgischen Implantataustausch. Dies erklärt die plötzliche Zunahme der Länge der Wirbelsäule, die in der Abbildung als Überschreiten der blauen Linie gegenüber der roten Linie sichtbar wird. (Figure 4 aus Lorenz et al. 2018).

\subsection{Erwarteter Implantatauszug im Vergleich zur tatsächlich gemessenen Implantatlänge (beide Implantate)}

Nach Auswertung von 723 Röntgenbildern, die nach jeder erfolgten Verlängerung angefertigt worden waren, konnte festgestellt werden, dass die tatsächliche Distraktion gegenüber der beabsichtigten für beide MAGEC®-Stabsysteme zusammen 94,4\% betrug. Das Verhältnis der erwarteten und erreichten Stablänge ist graphisch in der nachfolgenden Abbildung dargestellt (Abbildung 9). Hier zeigt sich stets eine Abweichung der blauen (erwarteter Auszug) zur roten (tatsächlicher Auszug) Linie, die immer etwas unterhalb liegt. 
Die maximale Abweichung voneinander zeigen beide Kurven nach vierzehn Verlängerungen mit 7,57 mm (89,2\%). Des Weiteren lässt sich aus der Abbildung 9 entnehmen, dass, wie auch für jeden Stab einzeln betrachtet (s. Abbildungen 11, 12), die Stabsysteme im Mittel nach jeder erfolgten Verlängerung in ihrer Länge weiter ausgezogen sind. Abbildung 10 gibt dazu die Anzahl der Patienten als graue Balken im Verhältnis zu den einzelnen erfolgten Verlängerungsprozeduren wieder. Die grünen Balken liefern einen Überblick über die Anzahl ausgetauschter MAGEC®- Stabsysteme. Während des gesamten Zeitraumes wurden 22 Stabsysteme ausgetauscht, die höchste Rate lag dabei mit einem absoluten Wert von fünf nach elf erfolgten Verlängerungseingriffen.

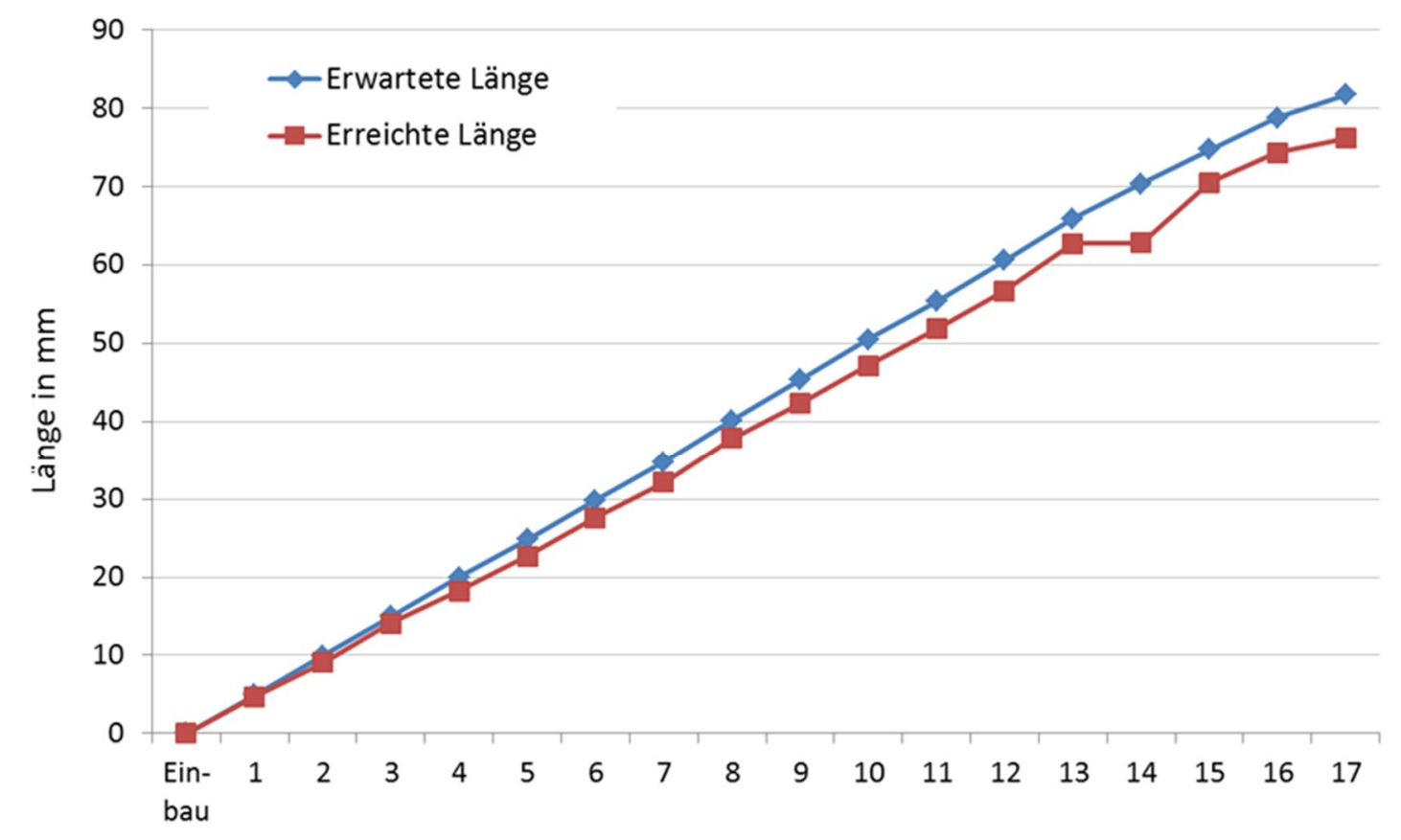

Anzahl der Verlängerungen

Abbildung 9: Verlängerung beider Implantate. Die erwartete (blau) und die erreichte (rot) Länge in Millimetern während der Therapie mit magnetisch gesteuerten Wirbelsäulenimplantaten mit maximal 17 Verlängerungen. 802 Röntgenbilder wurden ausgewertet. Die erreichte Distraktionslänge lag konstant unterhalb der gewünschten Länge, die Abweichung war jedoch moderat. Im Durchschnitt betrug die tatsächliche Distraktion gegenüber der beabsichtigten Distraktion 94,4\%. (Figure 2 aus Lorenz et al. 2018). 


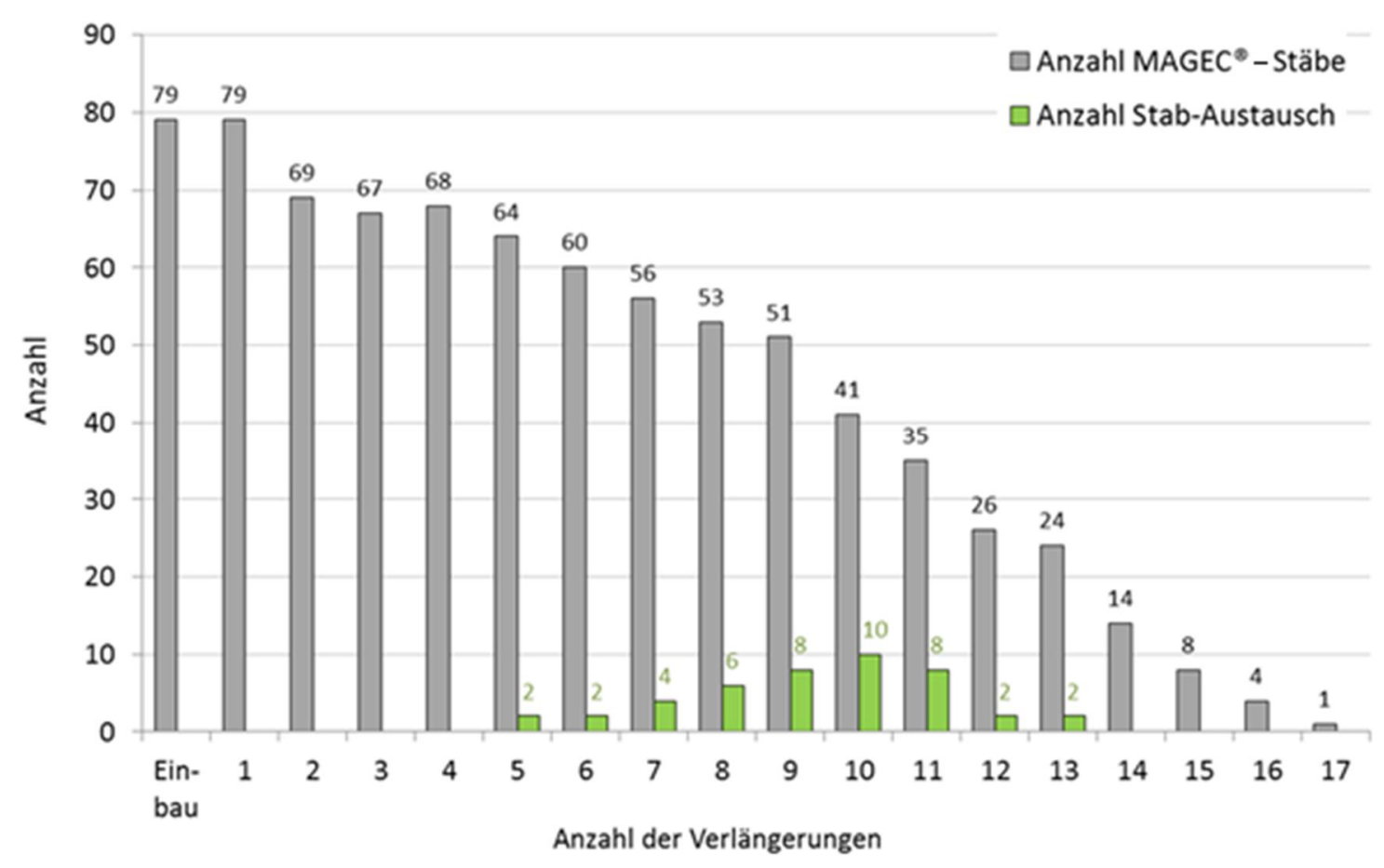

Abbildung 10: Anzahl der Patienten und Stabwechsel. Die grauen Balken stellen die Anzahl der Patienten dar, die für diese Studie für jede Verlängerung beobachtet wurden. Die grünen Balken zeigen die Anzahl der chirurgischen Stabaustausch-Eingriffe während der Behandlungsperiode.

\subsubsection{Unterschied zwischen dem konkaven und konvexen Implantat}

Die nachfolgend angeführten Abbildungen 11 und 12 verdeutlichen jeweils das Verhältnis der erwarteten Stabverlängerung im Vergleich zur retrospektiv, anhand von insgesamt 412 bzw. 390 (konkav/konvex) ermittelten, tatsächlichen Längenänderung des entsprechenden Implantats. Dazu wurde während der einzelnen Verlängerungseingriffe unter graphischer Berücksichtigung der Patientenanzahl (grauer Balken) die durchschnittliche erreichte (rot) und erwartete (blau) Auszuglänge des konkaven und konvexen Stabsystems in Millimeter $(\mathrm{mm})$ aufgetragen. Wieder zeigt sich eine stetige Abweichung der erreichten Auszuglänge (rote Linie) zur erwarteten (blaue Linie). Der Abstand hält sich für jedes Stabsystem einzeln betrachtet im moderaten Rahmen, zeigt sich jedoch im direkten Vergleich beim konvexen Stabsystem insgesamt über eine größere Anzahl an Verlängerungsprozeduren geringer. Die maximale Differenz zu Ungunsten des tatsächlichen Auszugs liegt auch hier für beide Stabsysteme zum Zeitpunkt der vierzehnten Verlängerung und beträgt absolut 8,79 $\mathrm{mm}$ (konkaves Implantat) bzw. 7,57 mm (konvexes Implantat). Folgend zeigt sich wieder eine Annäherung auf 3,93 mm bzw. 4,21 mm. Erfolgte Austausch- Operationen der MAGEC®Stäbe bei Erreichen der maximalen Auszuglänge oder bei operativ zu versorgenden Komplikationen wurden graphisch als grüne Balken berücksichtigt. 


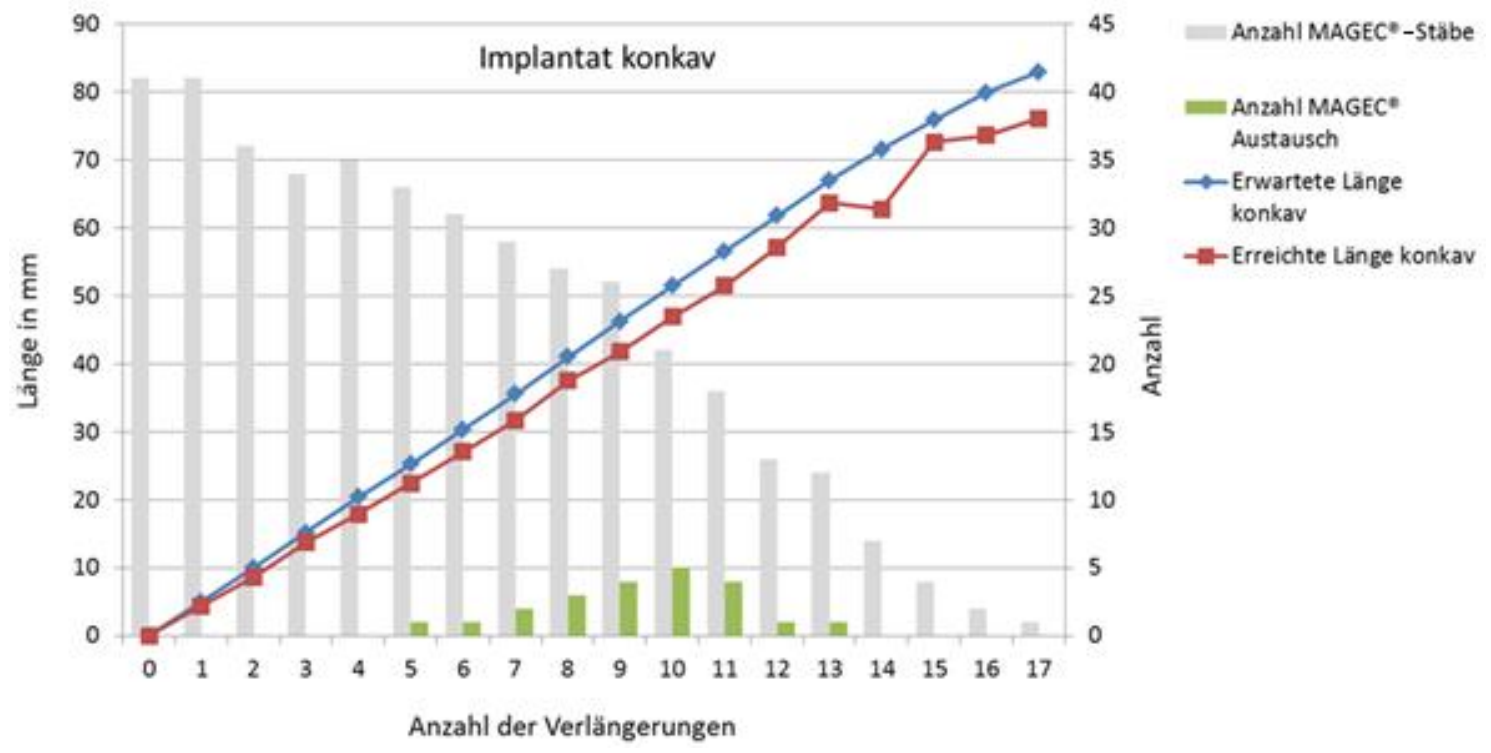

Abbildung 11: Verlängerung konkaves Implantat. Die erwartete (blau) und die erreichte (rot) Länge der konkaven Seite der skoliotischen Kurve während der Therapie mit magnetisch gesteuerten Wirbelsäulenimplantaten mit maximal 17 Verlängerungen. Auf Röntgenaufnahmen wurden 412 Stäbe bewertet. Die grauen Balken stellen die Anzahl der Patienten dar, die für diese Studie für jede Verlängerung beobachtet wurden. Die grünen Balken zeigen die Anzahl der chirurgischen Stabaustausch-Eingriffe während der Behandlungsperiode an. Die erreichte Distraktionslänge war konstant unterhalb der gewünschten Länge, aber die Abweichung über den ganzen evaluierten Zeitraum gemittelt war moderat $(3,3 \%)$.

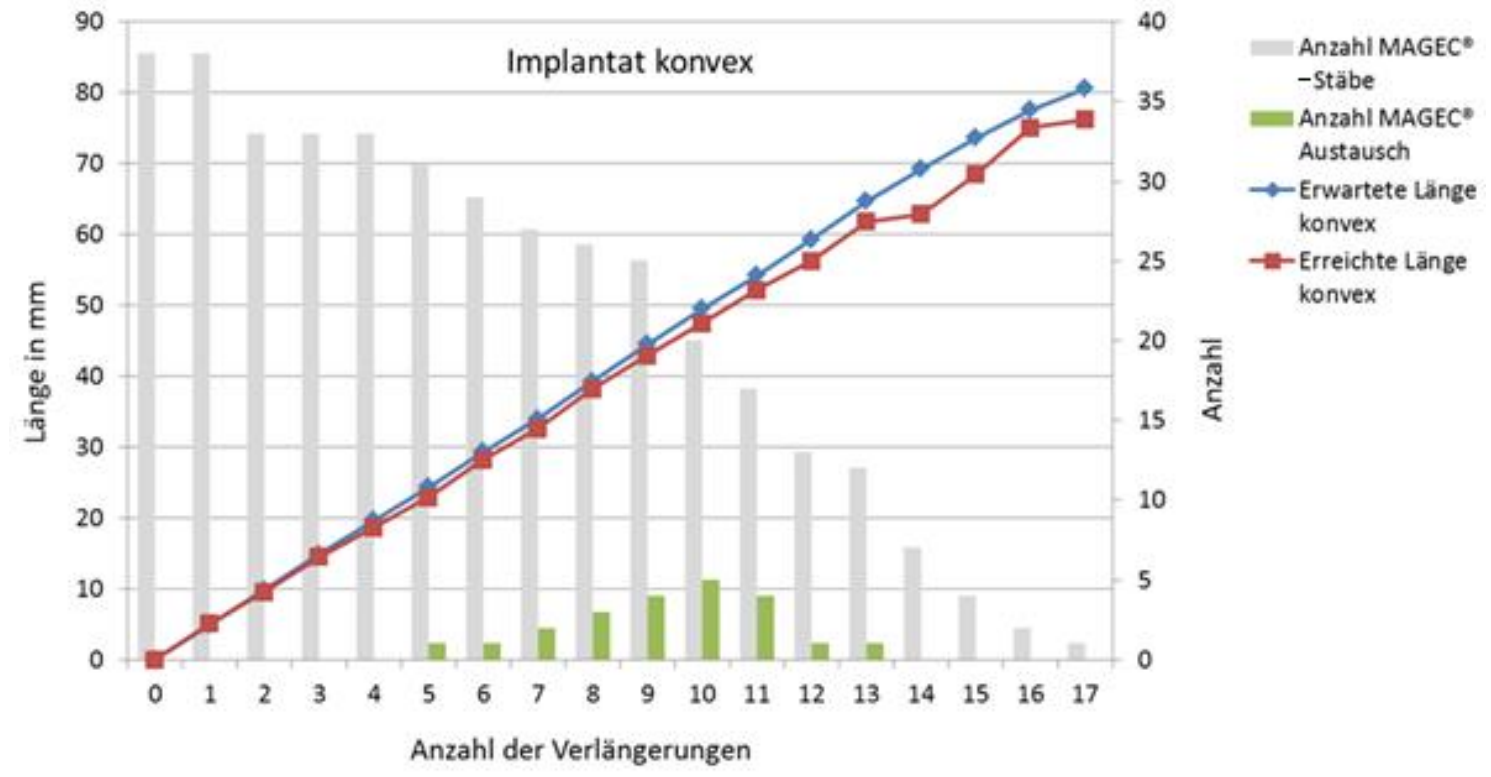

Abbildung 12: Verlängerung konvexes Implantat. Die erwartete (blau) und die erreichte (rot) Länge der konvexen Seite der skoliotischen Kurve während der Therapie mit magnetisch gesteuerten Wirbelsäulenimplantaten mit maximal 17 Verlängerungen. Auf Röntgenaufnahmen wurden 390 Stäbe bewertet. Die grauen Balken stellen die Anzahl der 
Patienten dar, die für diese Studie für jede Verlängerung beobachtet wurden. Die grünen Balken zeigen die Anzahl der chirurgischen Stabaustausch-Eingriffe während der Behandlungsperiode an. Die erreichte Distraktionslänge war konstant unterhalb der gewünschten Länge, aber die Abweichung über den ganzen beobachteten Zeitraum gemittelt war moderat $(5,8 \%)$.

\subsection{Komplikationen}

Während des gesamten Zeitraumes der Therapie (inklusive Implantation des MAGEC®Konstrukts) erfolgten 802 Interventionen. Hierbei traten insgesamt 35 Komplikationen auf, was einer Komplikationsrate von 4,4\% entspricht. Diese betrafen 19 der 41 in der Studie berücksichtigten Kinder (entspricht 46,3\%).

An allgemeinen Komplikationen konnten Dislokationen ( $n=4)$, Stabausbrüche ( $n=4)$, Bruch vom Beckenhaken $(n=2)$, Implantatbruch $(n=1)$ und das Auftreten von postoperativen Druckulzera/ Dekubiti $(n=3)$ verzeichnet werden. Zudem kam es in einem Fall zum Durchrutschen des Stabes durch die Muffe.

An Implantat-assoziierten Komplikationen kam es in 13 Fällen (1,6\%) zum fehlenden Ausfahren des Stabsystems, in drei Fällen zum Knacken/ Durchdrehen (0,37\%) und in einem Fall $(0,13 \%)$ zum unbeabsichtigten mit Ausfahren des anderen Stabes während der Verlängerung des gewünschten. Des Weiteren traten Implantatinfektionen (0,4\%) auf.

Bei Betrachtung des Verhältnisses von BMI und Art der Komplikation fiel auf, dass insbesondere ein hoher BMI ( $>90$. Perzentile) mit einem erhöhten Risiko des Auftretens eines fehlenden Ausfahrens des Stabsystems korrelierte. Entsprechend konnte genanntes Implantatversagen in 76,9\% der Fälle bei Kindern mit einem deutlich erhöhten BMI dokumentiert werden. Bei deutlichem Untergewicht (<3. Perzentile) kam es hingegen gehäuft zum unbeabsichtigten beidseitigen Stabauszug sowie zur Entstehung von Dekubiti/ Druckulcerationen (67\% der Fälle). Implantatinfektionen traten sowohl bei Kindern mit Über- als auch Untergewicht auf.

In der nachfolgenden Abbildung 13 sind Anzahl und Art der Komplikationen in Relation zum BMI zusammenfassend aufgeführt. 


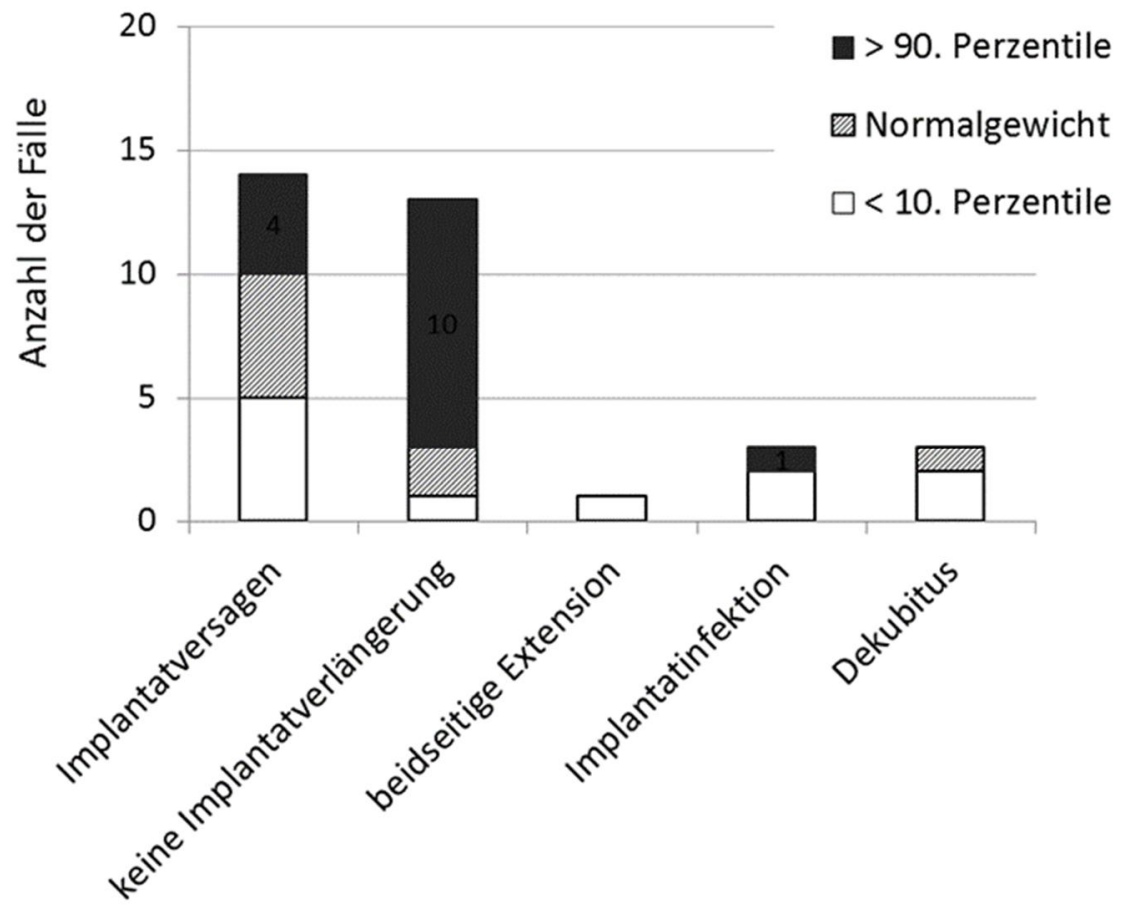

Abbildung 13: Komplikationen in Relation zum BMI. Anzahl der Komplikationen während der Therapie mit magnetisch gesteuerten Wirbelsäulen-Implantaten. In 802 Verfahren wurden 35 Komplikationen beobachtet (Komplikationsrate von 4,4\%). Komplikationen waren Implantatversagen $(n=15)$, fehlende Implantatverlängerung $(n=13)$, Implantatinfektion ( $n=3)$, Dekubitus $(n=3)$ und beidseitige Extension $(n=1)$. Daten zum BMI (Body-Mass-Index) sind ebenfalls enthalten. Der schwarze Balken zeigt einen BMI über dem 90. Perzentil, der graue Balken zeigt normales Gewicht an und der weiße Balken zeigt untergewichtige Kinder unter dem 10. Perzentil. (Figure 3 aus Lorenz et al. 2018).

\subsection{Korrelation des röntgenologisch ermittelten Implantatauszuges und des Cobb-Winkels bei Kindern mit Komplikationen}

In der nachfolgenden Abbildung 14 sind die Mittelwerte und Standardabweichungen des Cobb-Winkels präoperativ zu postoperativ für Kinder mit Komplikationen (rote Balken) und Kindern ohne Komplikationen (graue Balken) aufgeführt. Hierbei wird deutlich, dass sich dieser in beiden Personengruppen gleichermaßen postoperativ reduziert hat. Ferner zeigte sich auch der Implantatauszug bei Kindern mit und ohne Komplikationen nahezu identisch zuverlässig. 


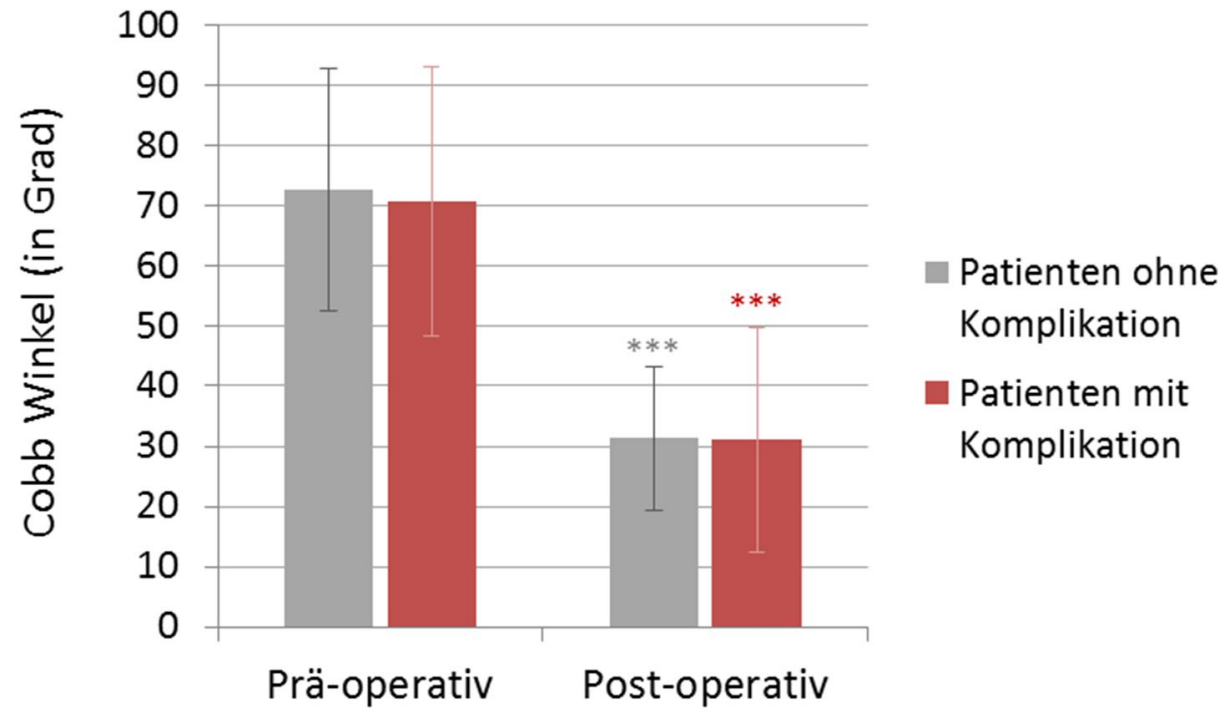

Abbildung 14: Veränderung des Cobb-Winkels in Grad präoperativ zu postoperativ für Kinder mit Komplikationen (rote Balken) und ohne (graue Balken), getrennt betrachtet. Der Cobb-Winkel zeigt sich in beiden Personengruppen im Mittel postoperativ gleichermaßen deutlich reduziert.

\subsection{Unbeabsichtigtes Implantat-Ein- oder Ausfahren zwischen den Verlängerungen}

Zur Beurteilung der Implantat-Stabilität im Sinne eines stabilen Längenzustandes der Implantate (konkav/ konvex) zwischen den einzelnen Verlängerungsprozeduren, wurden die Längen dieser sowohl nach als auch vor jedem erneuten Eingriff bestimmt.

Abbildung 15 zeigt die Veränderungen des Implantatzustands für den konkaven und konvexen MAGEC®-Stab zwischen den Verlängerungen in Millimetern (mm). Dargestellt ist der jeweilige Median (blaue Linie) und die Streuung als Interquartilsabstand Q1-Q3 (hellblauer Kasten). Der Median (mittlere Wert) liegt hierbei für das konkave Implantat bei $(-0,026)$ und für das konvexe System bei $(-0,027)$. Die schwarzen Linien deuten den Minimalund Maximalwert an.

Zusammenfassend betrachtet konnte keine signifikante Veränderung nach der letzten oder vor der nächsten Verlängerung beobachtet werden. Somit zeigte sich das Stabsystem in $79,8 \%$ der Fälle in einem stabilen Zustand. Als ungewollte Veränderung wird sowohl ein unbeabsichtigtes Wiedereinfahren als auch weiteres Ausfahren der Stabsysteme verstanden und statistisch als Längenabweichung kleiner als 5\% zwischen zwei Verlängerungen definiert. Entsprechend ergaben sich in 20,2\% der Fälle Abweichungen größer 5\% jedoch ohne den bildmorphologischen Nachweis der ungewollten Distraktion oder Retraktion. Somit scheint das Implantat in den Monaten zwischen den Eingriffen in seinem Zustand stabil zu sein. 


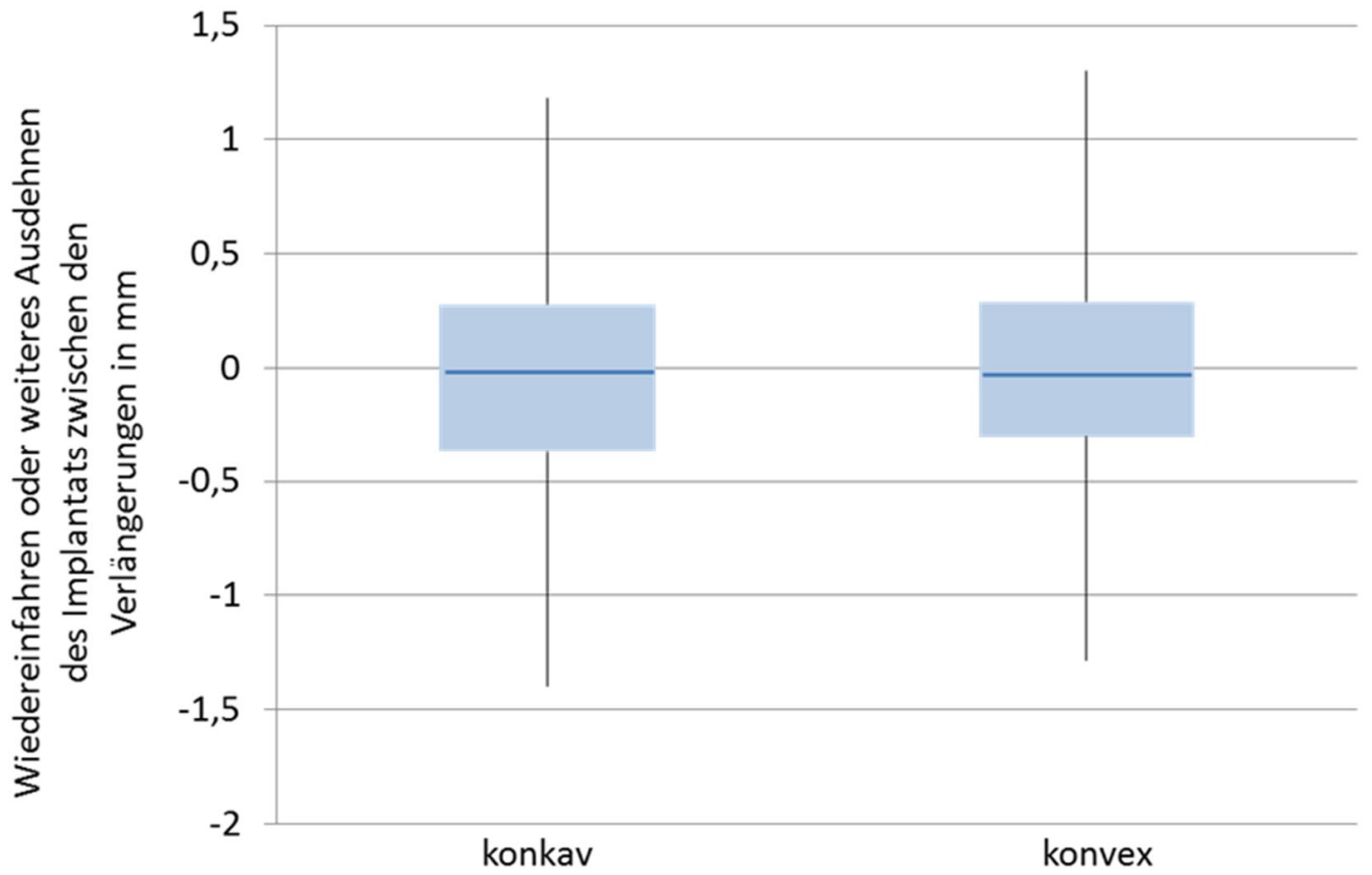

Abbildung 15: Stabilität des Implantatsystems. Änderungen der Implantatlänge zwischen den Distraktionseingriffen in $\mathrm{mm}$. Die blaue Linie stellt den Median dar, während die blaue Box den Interquartilabstand zwischen dem unteren Quartil (Q1; 25\%) und dem oberen Quartil (Q3; 75\%) anzeigt. Die schwarzen Linien beschreiben die minimalen und maximalen Werte. Beide magnetisch gesteuerten Stäbe (konkav und konvex) konnten zwischen den Eingriffen stabil gehalten werden und wurden nicht unbeabsichtigt zurückgezogen oder weiter abgelenkt.

\subsection{Messgenauigkeit / Inter-Observer}

Die Daten wurden größtenteils (zu etwa 80\%) von zwei voneinander unabhängigen Untersuchern erhoben. Die statistische Analyse wurde anhand der Mittelwerte aus den beiden Einzelwerten durchgeführt. Die Messgenauigkeit zwischen den beiden Untersuchern konnte durch die Analyse zur Inter-Observer-Abweichung beurteilt werden. Beispielhaft für die Messung des Auszugs des konvexen MAGEC®-Stabs wurde die Varianz untersucht, wobei kein signifikanter Unterschied der Ergebnisse zwischen den beiden Untersuchern bestand (Abbildung 16). Die Abweichung betrug im Durchschnitt 0,28 $\mathrm{mm}$ pro gemessenen Zeitpunkt. 


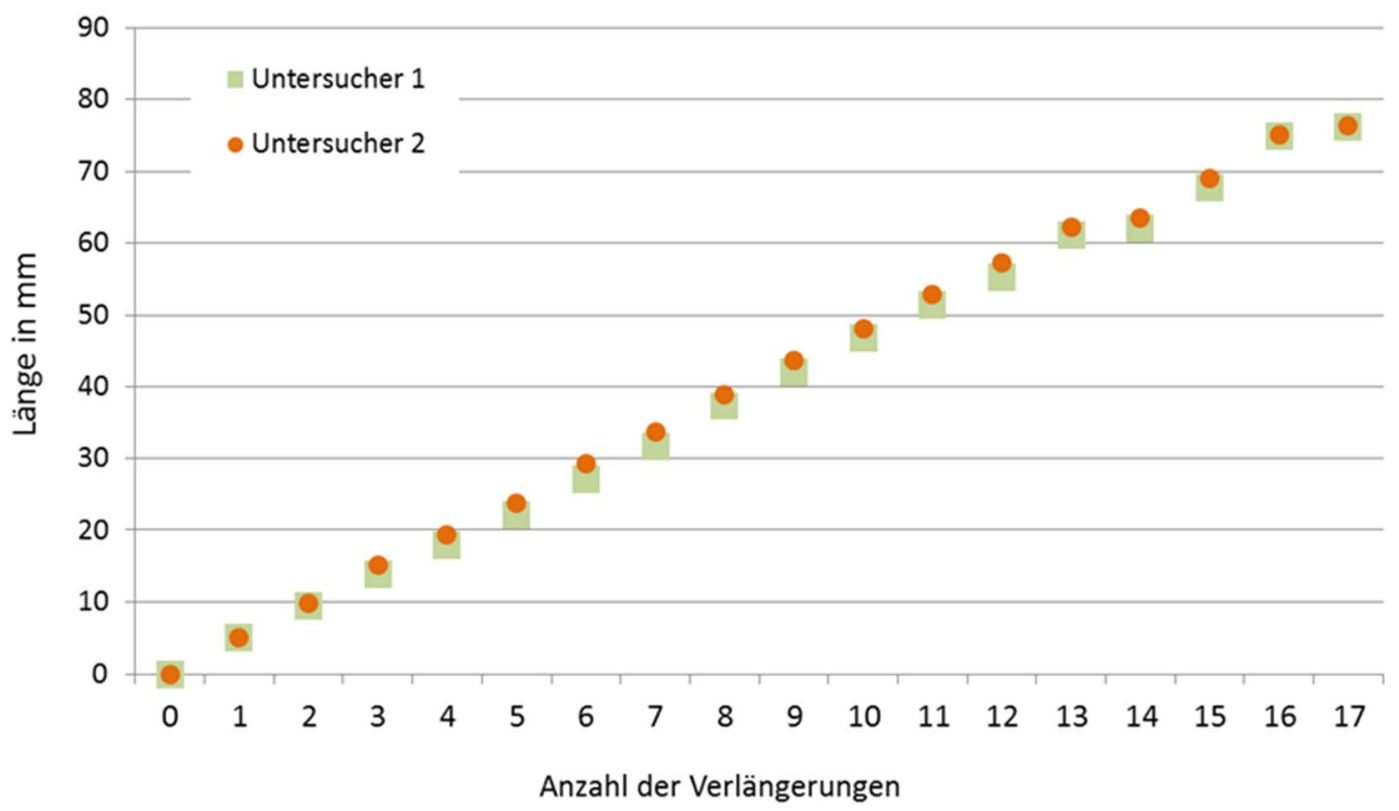

Abbildung 16: Varianz zwischen den beiden Untersuchern anhand des konvexen Stabauszuges (in $\mathrm{mm}$ ). 


\section{Diskussion}

Wirbelsäulendeformitäten im Kindesalter zeigen häufig einen progredienten Verlauf, welcher oftmals eine frühzeitige operative Versorgung erfordert. Goldstandard der heutigen Therapie stellen mitwachsende, nicht- fusionierende Implantate dar, die neben der Korrektur der Wirbelsäulendeformität das Wirbelsäulen- und somit auch Brustkorbwachstum ermöglichen, um ansonsten drohende körperliche Komplikationen, wie zum Beispiel die Ausbildung eines Thorax-Insuffizienz-Syndroms infolge eines eingeschränkten Lungenwachstums, respiratorische Insuffizienz, kosmetische und funktionelle Beeinträchtigungen, zu verhindern (Kwan et al. 2017; La Rosa et al. 2017). Bisher verwendete Implantatsysteme (z. B. growing rods, VEPTR) erfordern regelmäßige operative Längenanpassungen in Allgemeinanästhesie, meist in sechsmonatigen Intervallen. Neben der psychologischen Beeinträchtigung konnten verschiedene Komplikationen, wie bakterielle Implantatbesiedelung (Plaass et al. 2016; Wagner et al. 2018) sowie Autofusion und Ossifikationen (Groenefeld und Hell 2013; Zivkovic et al. 2014), nachgewiesen werden.

Mit Einführung von extern gesteuerten magnetischen wirbelsäulenaufrichtenden Implanten, den sogenannten magnetically controlled growing rods (Cheung et al. 2012), konnten die Anzahl der Operationen sowie die Komplikationsrate vermindert werden (Lorenz et al. 2017; Lorenz et al. 2018). Seither findet sich eine wachsende Anzahl an nachfolgenden Untersuchungen in der Literatur, die die Wirksamkeit, Effektivität und Sicherheit dieser magnetischen Teleskopimplantate belegen (Akbarnia et al. 2013; Hosseini et al. 2016; La Rosa et al. 2017; Lorenz et al. 2017). Besonders für Patienten mit erhöhtem Narkoserisiko, wie beispielsweise Patienten mit spinaler Muskelatrophie, konnte durch die Reduktion von Operationen eine wesentliche Verbesserung erreicht werden (Lorenz et al. 2017).

Neben der vergleichenden Analyse von Behandlungseffektivität und Komplikationsraten (Hickey et al. 2014; Doany et al. 2017) liegt ein Diskussionsfokus bei der Anwendung von magnetically controlled growing rods darin, dass die auf dem Display des externen Verlängerungsgerätes erscheinende Strecke nicht der reell im Körper vorgenommenen Verlängerung entsprechen muss. Eine Rückkoppelung von dem im Körper durchgeführten Prozess zur externen Einheit ist aktuell nicht vorhanden. So ist es beispielsweise möglich, dass durch das fehlerhafte Platzieren des external remote controller (ERC) zwar eine scheinbare Verlängerung an den Implantaten vorgenommen wird, welche aber wegen der Fehlplatzierung nicht am Implantat stattfindet. Rolton et al. konnten nur eine durchschnittliche Distraktion von 33\% der gewünschten Länge ermitteln (Rolton et al. 2016).

In der vorliegenden Untersuchung wurde bei einem Kollektiv von 41 Patienten radiologisch anhand von 802 Röntgenaufnahmen die reell erfolgte Distraktion der magnetically controlled growing rods mit der auf dem Display durchgeführten Verlängerung korreliert. 


\subsection{Vergleichende Analyse von erwarteter zur tatsächlichen Auszuglänge des MAGEC®- Stabsystems}

Anhand des untersuchten Patientenkollektivs konnte, sowohl für jeden MAGEC®-Stab einzeln als auch für beide Stabsysteme zusammen betrachtet, ein zuverlässiges Ausfahren des Systems nach jeder Distraktion nachgewiesen werden. Insgesamt zeigte sich stets eine jedoch nur moderate Diskrepanz zwischen der tatsächlichen Auszuglänge im Vergleich zur erwarteten. Über den mittleren Behandlungszeitraum von 32,6 Monaten erfolgten im Durchschnitt neun Distraktionen pro Patient, woraus sich eine Gesamtanzahl von 802 Interventionen (inklusive der 41 erfolgten Systemimplantationen) ergab. Die anhand der röntgenologischen Bildaufnahmen ermittelte durchschnittliche tatsächliche Auszuglänge beider Stabsysteme ergab im Vergleich zur erwarteten Auszuglänge ein Verhältnis von 94,4\%. Die statistische Analyse jedes Stabsystems einzeln, ergab dabei keine signifikante Abweichung vom Gesamtergebnis. Bereits Cheung und Kollegen beschrieben 2012 anhand zweier mittels MAGEC®-System behandelter Patienten eine Abweichung der mittleren von der tatsächlichen Längenänderung der Metallstabsysteme pro erfolgter Distraktion (Patient 1: 1,4 mm/ 2,3 mm; Patient 2: links 1,7 mm/ 2,1 mm; rechts 1,9 mm/ 2,0 mm) (Cheung et al. 2012). Eine ähnliche Differenz beschrieben La Rosa et al. anhand von zehn mittels MAGEC behandelter Patienten. Hier konnte im Durchschnitt ein Missverhältnis von 2,1 $\mathrm{mm}$ (tatsächlich) zu 3,0 mm (erwartet) pro Behandlungseinheit dokumentiert werden (La Rosa et al. 2017). Ferner berichteten andere Autoren von im Vergleich zu unserem Ergebnis deutlich niedrigeren Distraktionsraten von nur 33\% (Rolton et al. 2016) , 32\% (La Rosa et al. 2017) und 45,5\% (Lebon et al. 2016). Die dazu aktuellsten Ergebnisse präsentierten hierzu Gilday et al. 2018, die ein Verhältnis der tatsächlichen zur erwarteten Auszuglänge von 86\% feststellten (Gilday et al. 2018). Die doch erheblichen Abweichungen müssen vor dem Hintergrund unterschiedlich großer, heterogener Patientenkollektive, unterschiedlicher Beobachtungszeiträume, der verwendeten MCGR-Generation und der verwendeten Messmethode (röntgenologisch vs. sonographisch vs. fluoroskopisch) gesehen werden.

Zum Zeitpunkt der erfolgten 17. Verlängerungsprozedur konnte eine Differenz zwischen tatsächlicher und erwarteter Distraktionslänge von $76,19 \mathrm{~mm}$ zu 81,75 mm ermittelt werden. Die höchste Diskrepanz von 89,2\% zeigte sich zwischen genannten Größen nach 14 Systemverlängerungen (nach durchschnittlich 4,2 Jahren der Behandlung).

Als Ursache der Abweichung von tatsächlicher zur erwarteten Auszuglänge der MAGEC®Stäbe sind, wie bereits in der verfügbaren Literatur bereits diskutiert und erstmals durch Sankar et al. 2011 beschrieben, ein mit zunehmender Anzahl an Verlängerungsprozeduren bedingter Effizienzverlust der Distraktion (,law of diminishing returs"). Grundlage beschriebenen Phänomens bilden die Idee der zunehmenden Steifigkeit der Wirbelsäule und umliegenden Gewebes sowie Autofusionen durch rezidivierende Manipulationen (Sankar et al. 2011). In der vorliegenden Studie konnte dieses Phänomen nicht beobachtet werden. Möglicherweise könnte dies darin bergründet sein, dass der Großteil unserer Patienten 
(30/41) eine neuromuskuläre Skoliose, genauer eine spinale Muskelatrophie (SMA), als Grunderkrankung aufwiesen. Bei genanntem Erkrankungsbild ließ sich eine Assoziation zu einer erhöhten spinalen Flexibilität und einem damit höherem Korrekturpotential herstellen (Lorenz et al. 2017). Des Weiteren könnten die hier verwendete Wirbelsäulen-ferne Rippenzu Beckenverankerung (Hell et al. 2018) sowie die kurzen Intervalle zwischen den Distraktionen von drei Monaten einen möglichen positiven Einfluss auf das Ergebnis genommen haben.

Ferner konnte bei fehlendem Unterschied des Behandlungsergebnisses zwischen dem mit VEPTR-System vorbehandelten Patientenkollektiv und denen, die keine spinale Vorbehandlung erhalten habe, abgeleitet werden, dass sich genanntes Verfahren gleichermaßen als primäres und sekundäres Behandlungsverfahren eignet. Gleiches Ergebnis konnte bereits durch andere Autoren beobachtet werden (Hickey et al. 2014; Gilday et al. 2018).

Über den gesamten Behandlungszeitraum wurden 22 Stabsysteme ausgetauscht, die höchste Rate lag dabei mit einem absoluten Wert von fünf nach elf erfolgten Verlängerungseingriffen.

Neben der Anforderung an die von extern magnetisch zu kontrollierenden Wachstumsstäbe des zuverlässigen Ausfahrens, ergibt sich die Erwartung eines zwischen den einzelnen Verlängerungsintervallen in der Länge stabilen Systems. In 79,8\% der Fälle zeigte sich das Stabsystem in einem stabilen Zustand. Dieser wurde definiert als eine Abweichung der Stablänge zwischen zwei Distraktionen (nach erfolgter und vor erneuter Verlängerung) kleiner gleich 5\%. In den übrigen Fällen (20,2\%) zeigte sich eine Abweichung von mehr als $5 \%$, jedoch ohne eine bildmorphologische Tendenz der unbeabsichtigten Stabverlängerung oder Verkürzung.

\subsection{Vergleich von Stablänge und veränderte Wirbelsäulenlänge}

Die Bestimmung der spinalen Länge (s. Kapitel 2.4.3.2) zur objektiven Beurteilung des Behandlungserfolges zeigte eine strenge Korrelation zwischen der Auszuglänge des MAGEC ${ }^{\circledR}-$ Stabsystems und der spinalen Länge. Daraus kann abgeleitet werden, dass ein großer Anteil der Stabverlängerungen direkt auf das Längenwachstum der Wirbelsäule übertragen wird und hiermit schlussfolgernd einen positiven Einfluss auf die Körpergröße, einschließlich der Höhe des Oberkörpers nimmt. Die wenn auch geringe Diskrepanz lässt sich möglicherweise durch eine mit der Zeit zunehmenden Steifigkeit der Wirbelsäule erklären (Sankar et al. 2011). Damit lässt sich die Wirksamkeit und Effektivität der extern magnetisch $\mathrm{zu}$ kontrollierenden Implantaten, neben der Korrektur der Wirbelsäulendeformität das physiologische Wirbelsäulenwachstum zu ermöglichen, auch in unserem untersuchten Patientenkollektiv bestätigen. Dieses Ergebnis entspricht damit der vorliegenden Literatur, in der als $\mathrm{Maß}$ des Wirbelsäulenwachstums eine röntgenologische Vergrößerung der Distanz zwischen dem ersten und zwölften thorakalen Wirbelkörper (T1- 
T12), beziehungsweise dem ersten thorakalen und sakralen Wirbelkörper (T1-S1) herangezogen wird (Cheung et al. 2012; Akbarnia et al. 2013; Dannawi et al. 2013; Hickey et al. 2014; Cheung et al. 2017; La Rosa et al. 2017). Grundsätzlich wäre die Verwendung der Körpergröße und Armspannweite, entsprechend dem Vorgehen von Cheung und Kollegen (Cheung et al. 2012), als zusätzliche objektive Parameter des Wirbelsäulenwachstums in zukünftigen Untersuchungen als sinnvoll zu überdenken.

\subsection{Komplikationen}

Im untersuchten Patientenkollektiv konnten während eines mittleren Behandlungszeitraums von 32,6 Monaten (5 bis 57,3 Monate) und 802 erfolgten Interventionen insgesamt 35 Komplikationen notiert werden, was einer Komplikationsrate von 4,4\% entspricht. Diese betrafen dabei in unterschiedlicher Häufigkeit 19 der 41 berücksichtigten Patienten (entspricht 46,3\%). Komplikationsbedingte und somit zur Implantation zusätzliche Operationen waren bei elf Patienten erforderlich.

Im Vergleich zu anderen im Umfang (Patientenanzahl, Anzahl der Verlängerungen, Followup) ähnelnden Untersuchungen fiel die Komplikationsrate in der vorliegenden Studie durchschnittlich niedriger aus (Thakar et al. 2018). Zu berücksichtigen ist hierbei jedoch, dass die Anzahl der Komplikationen dabei nicht in Verhältnis zur Anzahl erfolgter Verlängerungen gesetzt worden ist. Kwan et al. beschrieben 2017 eine Rate an ungeplanten Operationen bei 14/30 mittels MAGEC®-Konstrukt behandelten Patienten (entspricht 46,7\%), am häufigsten infolge eines Implantatversagens bei fehlerhaften Ausfahren, Lockerung der Verankerung, Stabbruch und Infektion bedingt (Kwan et al. 2017).

An Komplikationen konnten in der Studie der Häufigkeit nach abnehmend Implantatversagen (15 Fälle), fehlendes Ausfahren des Stabsystems (13 Fälle), Implantatinfektionen (drei Fälle), Dekubitus (drei Fälle) und das unerwünschte beidseitige Ausfahren (ein Fall) beobachtet werden. Die Art sowie das Verteilungsmuster aufgetretener Komplikationen ähnelten dabei denen in der Literatur vorbeschriebenen (Thakar et al. 2018).

Partielle Distraktionsverluste, wie sie Akbarnia und Kollegen beschrieben, fanden sich im untersuchten Patientenkollektiv nicht (Akbarnia et al. 2013). Grund hierfür scheinen die damals verwendeten Systeme erster Generation (s. Kapitel 4.4) gewesen zu sein.

Zusammenfassend kann die niedrige Komplikationsrate (in Bezug auf die Gesamtanzahl erfolgter Interventionen) durch die geringere Invasivität des Verfahrens im Vergleich zu konventionellen mitwachsenden Systemen (z. B. growing rods, VEPTR) erklärt werden. Ferner wären womöglich das in der Studie gewählte Verfahren der Wirbelsäulen-fernen Verankerung mittels Komponenten des VEPTR-Systems sowie die gewählten Zeitintervalle von fünf Monaten zwischen Implantation und erster Verlängerung bzw. drei Monaten zwischen den einzelnen Distraktionen und dem Maß der Längenänderung von durchschnittlich $5 \mathrm{~mm}$ pro Sitzung als positive Einflussfaktoren zu diskutieren. Hierfür 
spricht insbesondere die durch Kwan et al. beschriebene Korrelation der Verlängerungsintervalle von zwei oder weniger Monaten mit dem Auftreten gehäufter unerwünschter Reoperationen (Risiko 71\% vs. 25\%) (Kwan et al. 2017).

Trotz unvermeidbarer Komplikationen ergibt sich bei der Verwendung von extern zu kontrollierenden magnetischen Implantaten ein deutlicher Benefit in Form einer gesteigerten Lebensqualität, allgemeiner Zufriedenheit und geringerer Kosten im Verlauf durch das Einsparen von Operationen (Cheung et al. 2012; Thakar et al. 2018).

\subsubsection{Komplikationen in Relation zum Body-Mass-Index}

Bei der näheren Betrachtung der im Studienkollektiv erfassten Komplikationen konnte eine enge Korrelation zwischen BMI und Art der Komplikation beobachtet werden. So fiel auf, dass bei Übergewicht, welches definiert ist als BMI größer der 90. Perzentile, gehäuft Implantatversagen in Form eines fehlenden Ausfahrens des Stabsystems beobachtet werden konnte. So wurde genannte Komplikation in 10/13 Fällen (entspricht 76,9 \%) bei Kindern mit Übergewicht dokumentiert. Ursächlich ist hierbei die durch das Vorliegen einer dickeren Gewebsschicht vergrößerte Distanz zwischen den interagierenden Magneten, die eine Abnahme des Magnetismus bewirkt. Dieser Zusammenhang wurde auch durch Cheung und andere Experten 2015 sowie 2017 beschrieben (Cheung et al. 2015; Cheung et al. 2017; Kwan et al. 2017).

Im Gegensatz dazu traten das unbeabsichtigte beidseitige Ausfahren des Stabsystems sowie die Entstehung postoperativer Dekubiti/ Druckulzerationen vermehrt bei deutlichem Untergewicht, genauer bei einem BMI kleiner der 3. Perzentile, auf. So zeigte sich in 67\% der Patienten mit Dekubiti deutliches Untergewicht. Analog obiger Erläuterung liegt genannte Korrelation in einer dünnen Schicht an Gewebe (fehlendem Weichteilmantel) begründet, die zu einer Verstärkung der Interaktion der Magneten führt. Ferner liegt das duale Stabsystem bei schmalem Habitus in engerer Lagebeziehung zueinander, was eine ungewollte Interaktion des externen Magneten mit beiden internen Aktuatoren begünstigt. Bei übrigen Komplikationen konnte kein Zusammenhang zum BMI hergestellt werden.

\subsection{Schlussfolgerungen, Limitationen und Ausblick}

In der vorliegenden Studie konnte an einem Patientenkollektiv ( $\mathrm{n}=41)$ mit Wirbelsäulendeformität unterschiedlicher Ätiologie über einen mittleren Beobachtungszeitraum von 32,6 Monaten zusammenfassend die Wirksamkeit der Behandlung mittels extern gesteuerten magnetischen wirbelsäulenaufrichtenden Implantaten sowohl als primäres als auch sekundäres Verfahren bestätigt werden. Mit einer Distraktionsrate von 94,4\% konnte in unserer Untersuchung eine höhere Korrelation zwischen erwartetem und tatsächlichem Stabauszug im Vergleich hierzu bereits vorliegender, verfügbarer Literatur gezeigt werden. Ein Unterschied mittels VEPTR-System 
vorbehandelter Patienten konnte hierbei nicht festgestellt werden. Entsprechend konnten eine enge Korrelation der Auszuglänge und spinalen Länge, als objektives Maß des Wirbelsäulenwachstums, sowie eine zwischen den Distraktionen bestehende Systemstabilität belegt werden.

Darüber hinaus ergab sich eine im Vergleich bekannter Ergebnisse niedrigere Komplikationsrate von 4,4\%, was mitunter als Einflussfaktor auf die zuverlässige Distraktion diskutiert werden sollte. An Risikofaktoren für das Versagen der Implantatverlängerung konnte lediglich ein hoher BMI ( $>$ 90. Perzentile) identifiziert werden. Positive Einflussnahme auf die geringe Komplikationsrate können das gewählte Zeitintervall von fünf Monaten zwischen Systemimplantation und erster Verlängerung, beziehungsweise drei Monaten zwischen den einzelnen Verlängerungsprozeduren und der gewählten Verlängerungsstrecke von $5 \mathrm{~mm}$ pro Eingriff nehmen. Genannte Paramater scheinen somit gut mit denen des physiologischen Wirbelsäulenwachstums übereinzustimmen.

Ferner ist von einer besseren gesundheitsbezogenen Lebensqualität infolge ambulanter Distraktionen und der Abwesenheit regelmäßiger, geplanter Operationen auszugehen. Dieser Aspekt wurde in der gegenwärtigen Untersuchung jedoch nicht beleuchtet.

Limitationen der vorliegenden Arbeit umfassen die Heterogenität des untersuchten Patientenkollektivs insbesondere hinsichtlich der Grunderkrankung, Vorbehandlung und unterschiedlichem Follow-up. Sinnvoll wäre hieran anschließend gleiches Patientenkollektiv und weitere den vordefinierten Einschlusskriterien entsprechende Patienten bis zu den definierten Endpunkten, bestensfalls der Spondylodese, nachzuuntersuchen, um den individuellen Erfolg der erwarteten zur tatsächlichen Distraktionslänge unter Korrelation der spinalen Länge und Dokumentation möglicher Komplikationen beurteilen zu können. Die Stärke der Studie liegt insbesondere in der verhältnismäßig großen Fallzahl, hohen Anzahl an Verlängerungsprozeduren und dem langen Follow-up von 32,6 Monaten.

Die oben angeführten erfolgreichen Studienergebnisse sind sicherlich auch im Hinblick bisheriger Verbesserungen des MAGEC®-Systems zu sehen. Diese umfassen neben der Veränderung des Fertigungsprozesses (Schweißprozesses) zur Reduktion von Stabbrüchen, das Einführen einer Halterplatte zur Stabilisierung des internen Magneten, die Längenanpassung der „aktiven“ Stabregion von wahlweise $70 \mathrm{~mm}$ oder $90 \mathrm{~mm}$ (Cheung et al. 2015; Kwan et al. 2017).

Zukünftig wäre die Entwicklung einer neuen Generation von extern gesteuerten magnetischen wirbelsäulenaufrichtigen Implantaten $\mathrm{zu}$ überdenken, die über einen zusätzlichen Rückkopplungsmechanismus verfügen, der eine nahezu vollständige Übertragung der gewünschten zur tatsächlichen Distraktionsstrecke sicherstellen ließe. 


\section{$5 \quad$ Zusammenfassung}

Progressive Wirbelsäulendeformitäten im Kindesalter benötigen häufig eine operative Therapie. Um neben der Korrektur der Wirbelsäulenverkrümmung das Wachstum der im Brustkorb befindlichen Lungen zu ermöglichen und somit die Entstehung eines ThoraxInsuffizienz-Syndroms zu verhindern, sind in den letzten Jahrzehnten verschiedene wachstumsfreundliche Wirbelsäulenimplantate entwickelt worden. Ein erheblicher Nachteil bisheriger Implantatsysteme liegt in regelmäßigen operativen Verlängerungen der Implantate und damit verbunden Komplikationen. Mit der Einführung von extern gesteuerten magnetischen wirbelsäulenaufrichtenden Implantaten, den sogenannten magnetically controlled growing rods (MCGR), konnte die Zahl der operativen Eingriffe für Kinder mit Wirbelsäulendeformität deutlich gesenkt werden.

In der vorliegenden Arbeit wurde das Verhältnis des tatsächlichen zum erwarteten Stabauszugs der uni- oder bilateral ( $n=3 ; n=38)$ implantierten MCGR durch Auswertung von 802 radiologischen Bildaufnahmen, die anhand eines Studienkollektivs $(n=41)$ mit Wirbelsäulendeformitäten unterschiedlicher Ätiologie über einen mittleren Beobachtungszeitraum von 32,6 Monaten angefertigt wurden, evaluiert. Berücksichtigt wurden zudem neben der veränderten Wirbelsäulenlänge das Auftreten von Komplikationen und Identifikation von Risikofaktoren, die zum Versagen der angestrebten Verlängerung führten. Die Stabverlängerungen erfolgten hierbei erstmals fünf Monate nach Implantation und fortlaufend in Drei-Monats-Abständen im ambulanten Rahmen.

Die Datenauswertung zeigte eine zuverlässige Wirksamkeit des Behandlungsverfahrens sowohl als primäres als auch sekundäres Verfahren in der Behandlung kindlicher Wirbelsäulendeformitäten. Bei einer Distraktionsrate von $94,4 \%$ konnte eine hohe Korrelation zwischen erwartetem und tatsächlichem (in vivo) Stabauszug belegt werden. Zudem wurde ein positiver Effekt der Stabverlängerungen auf das Längenwachstum der Wirbelsäule durch Zunahme der spinalen Länge dargestellt. Bei den im Beobachtungszeitraum von durchschnittlich 32,6 Monaten im Mittel erfolgten neun Distraktionen (1-17) pro Patient (entspricht 802 Interventionen) traten 35 Komplikationen auf, was einer Komplikationsrate von 4,4\% entspricht. Am häufigsten konnten hierbei neben einem Implantatversagen $(n=15)$ ein fehlender Auszug des Stabsystems $(n=13)$ dokumentiert werden. Letzteres korrelierte deutlich mit einem BMI $>90$. Perzentile $(76,9 \%$ der Fälle). Bei starkem Untergewicht ( $<3$. Perzentile) hingegen kam es vermehrt zum unbeabsichtigten beidseitigen Stabauszug sowie postoperativen Druckulcera/ Dekubiti (67\% der Fälle).

Fasst man zusammen, so konnte eine hohe Korrelation zwischen erwarteter und tatsächlicher Verlängerung von extern $\mathrm{zu}$ steuernden Implantaten für Kinder mit Wirbelsäulendeformität nachgewiesen werden. 


\section{$6 \quad$ Literaturverzeichnis}

Adipositas im Kindes- und Jugendalter. https://aga.adipositas-gesellschaft.de/index.php?id=39; Zugriff am 10.12.2017

Akbarnia BA, Emans JB (2010): Complications of growth-sparing surgery in early onset scoliosis. Spine (Phila Pa 1976) 35, 2193-2204

Akbarnia BA, Marks DS, Boachie-Adjei O, Thompson AG, Asher MA (2005): Dual growing rod technique for the treatment of progressive early-onset scoliosis: a multicenter study. Spine (Phila Pa 1976) $\underline{30}$, S46-57

Akbarnia BA, Mundis GM, Salari P, Yaszay B, Pawelek JB (2012): Innovation in growing rod technique: a study of safety and efficacy of a magnetically controlled growing rod in a porcine model. Spine (Phila Pa) 37, 1109-1114

Akbarnia BA, Cheung K, Noordeen H, Elsebaie H, Yazici M, Dannawi Z, Kabirian N (2013): Next generation of growth-sparing techniques: preliminary clinical results of a magnetically controlled growing rod in 14 patients with early-onset scoliosis. Spine $\underline{38}$, 665-670

Akbarnia BA, Pawelek JB, Cheung KMC, Demirkiran G, Elsebaie H, Emans JB, Johnston CE, Mundis GM, Noordeen H, Skaggs DL, et al. (2014): Traditional Growing Rods Versus Magnetically Controlled Growing Rods for the Surgical Treatment of Early-Onset Scoliosis: A Case-Matched 2-Year Study. Spine Deform 2, 493-497

Bekmez S, Dede O, Yazici M (2017): Advances in growing rods treatment for early onset scoliosis. Curr Opin Pediatr 29, 87-93

Bess S, Akbarnia BA, Thompson GH, Sponseller PD, Shah SA, El Sebaie H, Boachie-Adjei O, Karlin LI, Canale S, Poe-Kochert C, Skaggs DL (2010): Complications of growing-rod treatment for early-onset scoliosis: analysis of one hundred and forty patients. J Bone Joint Surg Am 22, 2533-2543

Bunnell WP (1988): The natural history of idiopathic scoliosis. Clin Orthop Relat Res $\underline{299}$, 20-25

Burnei G, Gavriliu S, Vlad C, Georgescu I, Ghita RA, Dughilă C, Japie EM, Onilă A (2015): Congenital scoliosis: an up-to-date. J Med Life $\underline{8}, 388-397$

Campbell RM Jr. (2013): VEPTR: past experience and the future of VEPTR principles. Eur Spine J 22 Suppl 2, S106-117

Campbell RM Jr., Hell-Vocke AK (2003): Growth of the thoracic spine in congenital scoliosis after expansion thoracoplasty. J Bone Joint Surg Am 모, 409-420

Campbell RM Jr., Smith MD, Mayes TC, Mangos JA, Willey-Courand DB, Kose N, Pinero RF, Alder ME, Duong HL, Surber JL (2003): The characteristics of thoracic insufficiency syndrome associated with fused ribs and congenital scoliosis. J Bone Joint Surg Am $\underline{85}$, 399-408

Campbell RM Jr., Smith MD, Mayes TC, Mangos JA, Willey-Courand DB, Kose N, Pinero RF, Alder ME, Duong HL, Surber JL (2004): The effect of opening wedge thoracostomy on thoracic insufficiency syndrome associated with fused ribs and congenital scoliosis. J Bone Joint Surg Am 무, 1659-1674 
Cheung JPY, Cahill P, Yaszay B, Akbarnia BA, Cheung KMC (2015): Special article: Update on the magnetically controlled growing rod: tips and pitfalls. J Orthop Surg (Hong Kong) $\underline{23}$, 383-390

Cheung JPY, Yiu KKL, Samartzis D, Kwan K, Tan B-B, Cheung KMC (2017): Rod lengthening with the magnetically controlled growing rod: factors influencing rod slippage and reduced gains during distractions. Spine (Phila Pa 1976) 43, E-399-E405

Cheung KM-C, Cheung JP-Y, Samartzis D, Mak K-C, Wong Y-W, Cheung W-Y, Akbarnia BA, Luk KD-K (2012): Magnetically controlled growing rods for severe spinal curvature in young children: a prospective case series. Lancet $\underline{379}, 1967-1974$

Cobb J (1948): Outline for the study of scoliosis. Instr Course Lect $\underline{5} 261-275$

Dannawi Z, Altaf F, Harshavardhana NS, El Sebaie H, Noordeen H (2013): Early results of a remotely-operated magnetic growth rod in early-onset scoliosis. Bone Joint J $\underline{95}, 75-80$

Dietz WH, Bellizzi MC (1999): Introduction: the use of body mass index to assess obesity in children. Am J Clin Nutr 므, 123S-125S

Dimeglio A (2001): Growth in pediatric orthopaedics. J Pediatr Orthop 21, 549-555

Doany ME, Olgun ZD, Kinikli GI, Bekmez S, Kocyigit A, Demirkiran G, Karaagaoglu AE, Yazici M (2017): Health-Related Quality of Life in Early-Onset Scoliosis Patients Treated Surgically: EOSQ scores in Traditional Growing Rod vs. Magnetically-Controlled Growing Rods. Spine (Phila Pa 1976) 43, 148-153

Figueiredo N, Kananeh SF, Siqueira HH, Figueiredo RC, Al Sebai MW (2016): The use of magnetically controlled growing rod device for pediatric scoliosis. Neurosciences (Riyadh) $\underline{21}, 17-25$

Flynn JM, Matsumoto H, Torres F, Ramirez N, Vitale MG (2012): Psychological dysfunction in children who require repetitive surgery for early onset scoliosis. J Pediatr Orthop $\underline{32}, 594$ 599

Gilday SE, Schwartz MS, Bylski-Austrow DI, Glos DL, Schultz L, O'Hara S, Jain VV, Sturm PF (2018): Observed Length Increases of Magnetically Controlled Growing Rods are Lower Than Programmed. J Pediatr Orthop $\underline{38}$, e133-e137

Groenefeld B, Hell AK (2013): Ossifications after vertical expandable prosthetic titanium rib treatment in children with thoracic insufficiency syndrome and scoliosis. Spine (Phila Pa 1976) $\underline{38}, \mathrm{E} 819-823$

Harrington PR (1962): Treatment of scoliosis. Correction and internal fixation by spine instrumentation. J Bone Joint Surg Am 44, 591-610

Hell AK, Hefti F, Campbell RM (2004): Behandlung der kongenitalen Skoliose mit dem Verticalexpandable-prosthetic-titanium-rib-Implantat. Orthopäde $\underline{33}$, 911-918

Hell AK, Groenefeld K, Tsaknakis K, Braunschweig L, Lorenz HM (2018): Combining Bilateral Magnetically Controlled Implants Inserted Parallel to the Spine With Rib to Pelvis Fixation: Surgical Technique and Early Results. Clin Spine Surg 31, 239-246

Hickey BA, Towriss C, Baxter G, Yasso S, James S, Jones A, Howes J, Davies P, Ahuja S (2014): Early experience of MAGEC magnetic growing rods in the treatment of early onset scoliosis. Eur Spine J 23 Suppl 1, S61-65 
Hosseini P, Pawelek J, Mundis GM, Yaszay B, Ferguson J, Helenius I, Cheung KM, Demirkiran G, Alanay A, Senkoylu A, et al. (2016): Magnetically controlled Growing Rods for Early-onset Scoliosis: A Multicenter Study of 23 Cases With Minimum 2 years Follow-up. Spine (Phila $\mathrm{Pa}$ 1976) $41,1456-1462$

Jenks M, Craig J, Higgins J, Willits I, Barata T, Wood H, Kimpton C, Sims A (2014): The MAGEC system for spinal lengthening in children with scoliosis: A NICE Medical Technology Guidance. Appl Health Econ Health Policy 12, 587-599

Karol LA (2011): Early definitive spinal fusion in young children: what we have learned. Clin Orthop Relat Res $\underline{469}, 1323-1329$

Kromeyer-Hauschild K, Wabitsch M, Kunze D, Geller F, Geiß HC, Hesse V, von Hippel A, Jaeger U, Johnsen D, Korte W, et al. (2001): Perzentile für den Body-mass-Index für das Kindesund Jugendalter unter Heranziehung verschiedener deutscher Stichproben. Monatsschr Kinderheilk 149, 807-818

Kwan KYH, Alanay A, Yazici M, Demirkiran G, Helenius I, Nnadi C, Ferguson J, Akbarnia BA, Cheung JPY, Cheung KMC (2017): Unplanned Reoperations in Magnetically Controlled Growing Rod Surgery for Early Onset Scoliosis With a Minimum of Two-Year Follow-Up. Spine (Phila Pa 1976) 42, E1410-E1414

La Rosa G, Oggiano L, Ruzzini L (2017): Magnetically Controlled Growing Rods for the Management of Early-onset Scoliosis: A Preliminary Report. J Pediatr Orthop 37, 79-85

Lebon J, Batailler C, Wargny M, Choufani E, Violas P, Fron D, Kieffer J, Accadbled F, Cunin V, De Gauzy JS (2016): Magnetically controlled growing rod in early onset scoliosis: a 30-case multicenter study. Eur Spine J 26, 1567-1576

Lorenz HM, Badwan B, Hecker MM, Tsaknakis K, Groenefeld K, Braunschweig L, Hell AK (2017): Magnetically Controlled Devices Parallel to the Spine in Children with Spinal Muscular Atrophy. JB JS Open Access 2, e0036

Lorenz HM, Braunschweig L, Badwan B, Groenefeld K, Hecker MM, Tsaknakis K, Grote J, Hell AK (2018): High Correlation Between Achieved and Expected Distraction Using Magnetically Controlled Growth Rods (MCGR) With Rib to Pelvis Fixation in Pediatric Spine Deformity. J Pediatr Orthop 39, E334-E338

Mundis GM, Pawelek JB, Nomoto EK, Hennessy MW, Yaszay B, Akbarnia BA (2016): Longitudinal Pilot Analysis of Radiation Exposure During the Course of Growing Rod Treatment for Early-Onset Scoliosis. Spine Deform 4, 55-58

Noonan KJ, Dolan LA, Jacobson WC, Weinstein SL (1997): Long-term psychosocial characteristics of patients treated for idiopathic scoliosis. J Pediatr Orthop 17, 712-717

Plaass C, Hasler CC, Heininger U, Studer D (2016): Bacterial colonization of VEPTR implants under repeated expansions in children with severe early onset spinal deformities. Eur Spine $\mathrm{J} \underline{25}, 549-556$

Poon S, Nixon R, Wendolowski S, Gecelter R, Chen YH, DiMauro J-P, Amaral T, Graver A, Grande DA (2017): A pilot cadaveric study of temperature and adjacent tissue changes after exposure of magnetic-controlled growing rods to MRI. Eur Spine J 26, 1618-1623

Ramirez N, Flynn JM, Serrano JA, Carlo S, Cornier AS (2009): The Vertical Expandable Prosthetic Titanium Rib in the treatment of spinal deformity due to progressive early onset scoliosis. J Pediatr Orthop B 18, 197-203 
Ridderbusch K, Rupprecht M, Kunkel P, Stücker R (2013): Nonfusionstechniken zur Behandlung der kindlichen Skoliosen. Orthopäde 42, 1030-1037

Ridderbusch K, Rupprecht M, Kunkel P, Hagemann C, Stücker R (2017): Preliminary Results of Magnetically Controlled Growing Rods for Early Onset Scoliosis. J Pediatr Orthop 37, e575-e580

Rolton D, Thakar C, Wilson-MacDonald J, Nnadi C (2016): Radiological and clinical assessment of the distraction achieved with remotely expandable growing rods in early onset scoliosis. Eur Spine J 25, 3371-3376

Sankar WN, Skaggs DL, Yazici M, Johnston CE, Shah SA, Javidan P, Kadakia RV, Day TF, Akbarnia BA (2011): Lengthening of dual growing rods and the law of diminishing returns. Spine (Phila Pa 1976) $\underline{36}$, 806-809

Sewell MD, Malagelada F, Wallace C, Gibson A, Noordeen H, Tucker S, Molloy S, Lehovsky J (2016): A Preliminary Study to Assess Whether Spinal Fusion for Scoliosis Improves Carer-assessed Quality of Life for Children With GMFCS Level IV or V Cerebral Palsy. J Pediatr Orthop $\underline{36}$, 299-304

SRS: „Early Onset" Skoliose. Operation: in situ Fusion der Wirbelsäule. SRS.org 2017. https://www.srs.org/german/patient_and_family/scoliosis/early_onset_scoliosis/treatme nt/in-situ.htm; Zugriff am 14.06.2017

Takaso M, Moriya H, Kitahara H, Minami S, Takahashi K, Isobe K, Yamagata M, Otsuka Y, Nakata Y, Inoue M (1998): New remote-controlled growing-rod spinal instrumentation possibly applicable for scoliosis in young children. J Orthop Sci $\underline{3}, 336-340$

Teoh KH, Winson DMG, James SH, Jones A, Howes J, Davies PR, Ahuja S (2016): Magnetic controlled growing rods for early-onset scoliosis: a 4-year follow-up. Spine J $\underline{16}$, S34-39

Thakar C, Kieser DC, Mardare M, Haleem S, Fairbank J, Nnadi C (2018): Systematic review of the complications associated with magnetically controlled growing rods for the treatment of early onset scoliosis. Eur Spine J 27, 2062-2071

Trobisch P, Suess O, Schwab F (2010): Idiopathic scoliosis. Dtsch Ärztebl Int 107, 875-883; quiz 884

Tübingen BMI-Rechner. https://www.medizin.unituebingen.de/kinderklinik/de/abteilungen/allgemeine-paediatrie-haematologieonkologie/sprechstunden/endokrinologie-adipositas/bmi-rechner/). Zugriff am 12.10.2017

Wagner L, Braunschweig L, Eiffert H, Tsaknakis K, Kamin D, D’Este E, Messer PK, Hell AK, Lorenz HM (2018): Detection of Bacteria Colonizing Titanium Spinal Implants in Children. Surg Infect (Larchmt) 19, 71-77

Watanabe K, Uno K, Suzuki T, Kawakami N, Tsuji T, Yanagida H, Ito M, Hirano T, Yamazaki K, Minami S, et al. (2013): Risk factors for complications associated with growing-rod surgery for early-onset scoliosis. Spine (Phila Pa 1976) $\underline{38}$, E464-468

Wilkins RM, Soubeiran A (2001): The Phenix expandable prosthesis: early American experience. Clin Orthop Relat Res $\underline{382}, 51-58$

Zivkovic V, Büchler P, Ovadia D, Riise R, Stuecker R, Hasler C (2014): Extraspinal ossifications after implantation of vertical expandable prosthetic titanium ribs (VEPTRs). J Child Orthop $\underline{8}, 237-244$ 


\section{Danksagung}

Mein ganz besonderer Dank gilt meiner Doktormutter Frau Prof. Dr. med. A.-K. Hell, die mir jederzeit sowohl fachlich als auch menschlich zur Seite stand und insbesondere durch anregende fachliche Diskussionen zum Erfolg meiner Dissertation beitrug.

Ebenso bedanke ich mich aufrichtig bei Frau Dr. rer. medic. Lena Braunschweig, die mich ebenfalls über die gesamte Zeit der Erstellung meiner wissenschaftlichen Arbeit durch ihr besonderes Wissen über statistische Auswertung und graphische Darstellung unterstützte und mich durchweg motivierte. 